\title{
ON A CONSTRUCTION FOR THE GENERATORS OF THE POLYNOMIAL ALGEBRA AS A MODULE OVER THE STEENROD ALGEBRA
}

\author{
NGUYỄN SUM
}

\begin{abstract}
Let $P_{n}$ be the graded polynomial algebra $\mathbb{F}_{2}\left[x_{1}, x_{2}, \ldots, x_{n}\right]$ with the degree of each generator $x_{i}$ being 1 , where $\mathbb{F}_{2}$ denote the prime field of two elements.

The Peterson hit problem is to find a minimal generating set for $P_{n}$ regarded as a module over the mod-2 Steenrod algebra, $\mathcal{A}$. Equivalently, we want to find a vector space basis for $\mathbb{F}_{2} \otimes_{\mathcal{A}} P_{n}$ in each degree $d$. Such a basis may be represented by a list of monomials of degree $d$.

In this paper, we present a construction for the $\mathcal{A}$-generators of $P_{n}$ and prove some properties of it. We also explicitly determine a basis of $\mathbb{F}_{2} \otimes_{\mathcal{A}} P_{n}$ for $n=5$ and the degree $d=15.2^{s}-5$ with $s$ an arbitrary positive integer. These results are used to verify Singer's conjecture for the fifth Singer algebraic transfer in respective degree.
\end{abstract}

\section{INTRODUCTION}

Denote by $P_{n}:=\mathbb{F}_{2}\left[x_{1}, x_{2}, \ldots, x_{n}\right]$ the polynomial algebra over the field of two elements, $\mathbb{F}_{2}$, in $n$ generators $x_{1}, x_{2}, \ldots, x_{n}$, each of degree 1 . This algebra arises as the cohomology with coefficients in $\mathbb{F}_{2}$ of a classifying space of an elementary abelian 2-group of rank $n$. Therefore, $P_{n}$ is a module over the mod-2 Steenrod algebra, $\mathcal{A}$. The action of $\mathcal{A}$ on $P_{n}$ is determined by the elementary properties of the Steenrod squares $S q^{i}$ and subject to the Cartan formula $S q^{k}(f g)=\sum_{i=0}^{k} S q^{i}(f) S q^{k-i}(g)$, for $f, g \in P_{n}$ (see Steenrod and Epstein [25]).

A polynomial $g$ in $P_{n}$ is called $h i t$ if it belongs to $\mathcal{A}^{+} P_{n}$, where $\mathcal{A}^{+}$is the augmentation ideal of $\mathcal{A}$. That means $g$ can be written as a finite sum $g=\sum_{j>0} S q^{j}\left(g_{j}\right)$ for suitable polynomials $g_{j} \in P_{n}$.

We study the Peterson hit problem of determining a minimal set of generators for the polynomial algebra $P_{n}$ as a module over the Steenrod algebra. Equivalently, we want to find a vector space basis for the quotient

$$
Q P_{n}:=P_{n} / \mathcal{A}^{+} P_{n}=\mathbb{F}_{2} \otimes_{\mathcal{A}} P_{n} .
$$

The problem was first studied by Peterson [17], Wood [36], Singer [23], and Priddy 20, who showed its relation to several classical problems in the homotopy theory. Then, this problem was investigated by Carlisle and Wood [3], Crabb and Hubbuck 4, Janfada and Wood [10, Kameko [1], Mothebe [14, Nam [15], Phuc and Sum [18, 19, Silverman [21], Silverman and Singer [22], Singer [24], Walker and Wood [35, Wood [37] and others.

2010 Mathematics Subject Classification. Primary 55S10; Secondary 55S05, 55T15.

Key words and phrases. Steenrod algebra, Peterson hit problem, algebraic transfer, polynomial algebra. 
The vector space $Q P_{n}$ was explicitly calculated by Peterson [17] for $n=1,2$, by Kameko [11] for $n=3$, and recently by us [26, 27] for $n=4$, unknown in general.

Let $G L_{n}$ be the general linear group over the field $\mathbb{F}_{2}$. This group acts naturally on $P_{n}$ by matrix substitution. Since the two actions of $\mathcal{A}$ and $G L_{n}$ upon $P_{n}$ commute with each other, there is an inherited action of $G L_{n}$ on $Q P_{n}$.

Denote by $\left(P_{n}\right)_{d}$ the subspace of $P_{n}$ consisting of all the homogeneous polynomials of degree $d$ in $P_{n}$ and by $\left(Q P_{n}\right)_{d}$ the subspace of $Q P_{n}$ consisting of all the classes represented by the elements in $\left(P_{n}\right)_{d}$. In [23], Singer defined the algebraic transfer, which is a homomorphism

$$
\varphi_{n}: \operatorname{Tor}_{n, n+d}^{\mathcal{A}}\left(\mathbb{F}_{2}, \mathbb{F}_{2}\right) \longrightarrow\left(Q P_{n}\right)_{d}^{G L_{n}}
$$

from the homology of the Steenrod algebra to the subspace of $\left(Q P_{n}\right)_{d}$ consisting of all the $G L_{n}$-invariant classes. It is a useful tool in describing the homology groups of the Steenrod algebra, $\operatorname{Tor}_{n, n+d}^{\mathcal{A}}\left(\mathbb{F}_{2}, \mathbb{F}_{2}\right)$. This transfer was studied by Boardman [1, Bruner, Hà and Hưng [2], Hưng 8], Chơn-Hà [6, 7, Minami [13, Nam [16], Hưng-Quỳnh [9], the present author [28] and others.

It was shown that the transfer is an isomorphism for $n=1,2$ by Singer in 23 and for $n=3$ by Boardman in [1]. However, for any $n \geqslant 4, \varphi_{n}$ is not a monomorphism in infinitely many degrees (see Singer [23, Hưng 8.) Singer made a conjecture that the algebraic transfer $\varphi_{n}$ is an epimorphism for any $n \geqslant 0$. This conjecture is true for $n \leqslant 3$. It can be verified for $n=4$ by using the results in 26, 27. The conjecture for $n \geqslant 5$ is an open problem.

In this paper, we present a construction for the $\mathcal{A}$-generators of $P_{n}$ and explicitly determine a basis of $\mathbb{F}_{2} \otimes_{\mathcal{A}} P_{n}$ for $n=5$ and the degree $d=15.2^{s}-5$ with $s$ an arbitrary positive integer. These results are used to study the fifth Singer algebraic transfer. We will show that Singer's conjecture for the fifth algebraic transfer is true in this case.

This paper is organized as follows. In Section 2, we recall some needed information on the weight vectors of monomials, the admissible monomials in $P_{n}$ and Singer's criterion on the hit monomials. In Sections 3, we present a construction for the $\mathcal{A}$-generators of $P_{n}$ and some properties of it. A basis of $Q P_{5}$ in the degree $d=15.2^{s}-5$ will be explicitly determined in Section 4. In Section 5, we show that Singer's conjecture for the fifth algebraic transfer is true in the internal degree $d$ by computing the space $\left(Q P_{5}\right)_{d}^{G L_{5}}$. Finally, in Section 6 we list all admissible monomials of the degree $d$ in $P_{5}$.

\section{Preliminaries}

In this section, we recall some needed information from Kameko 11 and Singer 24] on the weight vector of a monomial, the admissible monomials and Kameko's homomorphism.

\subsection{The weight vector of a monomial.}

Notation 2.1.1. We denote $\mathbb{N}_{n}=\{1,2, \ldots, n\}, \mathbb{J}=\left\{j_{1}, j_{2}, \ldots, j_{t}\right\} \subset \mathbb{N}_{n}$ and

$$
X_{\mathbb{J}}=X_{\left\{j_{1}, j_{2}, \ldots, j_{t}\right\}}=\prod_{j \in \mathbb{N}_{n} \backslash \mathbb{J}} x_{j} .
$$

In particular, $X_{\mathbb{N}_{n}}=1, X_{\emptyset}=x_{1} x_{2} \ldots x_{n}, X_{j}=x_{1} \ldots \hat{x}_{j} \ldots x_{n}, 1 \leqslant j \leqslant n$, and $X:=X_{n}=x_{1} x_{2} \ldots x_{n-1} \in P_{n-1}$. 
Let $\alpha_{i}(a)$ denote the $i$-th coefficient in dyadic expansion of a non-negative integer $a$. That means $a=\alpha_{0}(a) 2^{0}+\alpha_{1}(a) 2^{1}+\alpha_{2}(a) 2^{2}+\ldots$, for $\alpha_{i}(a)=0$ or 1 with $i \geqslant 0$. Let $x=x_{1}^{a_{1}} x_{2}^{a_{2}} \ldots x_{n}^{a_{n}} \in P_{n}$. Denote $\nu_{t}(x)=a_{t}, 1 \leqslant t \leqslant n$. Set

$$
\mathbb{J}_{i}(x)=\left\{t \in \mathbb{N}_{n}: \alpha_{t}\left(\nu_{i}(x)\right)=0\right\},
$$

for $i \geqslant 0$. Then, we have $x=\prod_{i \geqslant 0} X_{\mathbb{J}_{i}(x)}^{2^{i}}$.

Definition 2.1.2. For a monomial $x$ in $P_{n}$, define two sequences associated with $x$ by

$$
\begin{aligned}
\omega(x) & =\left(\omega_{1}(x), \omega_{2}(x), \ldots, \omega_{i}(x), \ldots\right), \\
\sigma(x) & =\left(\nu_{1}(x), \nu_{2}(x), \ldots, \nu_{n}(x)\right),
\end{aligned}
$$

where $\omega_{i}(x)=\sum_{1 \leqslant j \leqslant n} \alpha_{i-1}\left(\nu_{j}(x)\right)=\operatorname{deg} X_{\mathbb{J}_{i-1}(x)}, i \geqslant 1$. The sequences $\omega(x)$ and $\sigma(x)$ is respectively called the weight vector and the exponent vector of $x$.

Let $\omega=\left(\omega_{1}, \omega_{2}, \ldots, \omega_{i}, \ldots\right)$ be a sequence of non-negative integers. The sequence $\omega$ are called the weight vector if $\omega_{i}=0$ for $i \gg 0$.

The sets of all the weight vectors and the exponent vectors are given the left lexicographical order.

For a weight vector $\omega$, we define $\operatorname{deg} \omega=\sum_{i>0} 2^{i-1} \omega_{i}$. If there are $i_{0}=$ $0, i_{1}, i_{2}, \ldots, i_{r}>0$ such that $i_{1}+i_{2}+\ldots+i_{r}=m, \omega_{i_{1}+\ldots+i_{s-1}+t}=b_{s}, 1 \leqslant t \leqslant$ $i_{s}, 1 \leqslant s \leqslant r$, and $\omega_{i}=0$ for all $i>m$, then we write $\omega=\left(b_{1}^{\left(i_{1}\right)}, b_{2}^{\left(i_{2}\right)}, \ldots, b_{r}^{\left(i_{r}\right)}\right)$. Denote $b_{u}^{(1)}=b_{u}$. For example, $\omega=(3,2,2,2,1,1,0, \ldots)=\left(3,2^{(3)}, 1^{(2)}\right)$.

Denote by $P_{n}(\omega)$ the subspace of $P_{n}$ spanned by all monomials $y$ such that $\operatorname{deg} y=\operatorname{deg} \omega, \omega(y) \leqslant \omega$, and by $P_{n}^{-}(\omega)$ the subspace of $P_{n}$ spanned by all monomials $y \in P_{n}(\omega)$ such that $\omega(y)<\omega$. Denote by $\mathcal{A}_{s}^{+}$the subspace of $\mathcal{A}$ spanned by all $S q^{j}$ with $1 \leqslant j<2^{s}$.

Definition 2.1.3. Let $\omega$ be a sequence of non-negative integers and $f, g$ two polynomials of the same degree in $P_{n}$.

i) $f \equiv g$ if and only if $f-g \in \mathcal{A}^{+} P_{n}$.

ii) $f \equiv \equiv_{\omega} g$ if and only if $f-g \in \mathcal{A}^{+} P_{n}+P_{n}^{-}(\omega)$.

iii) $f \simeq{ }_{(s, \omega)} g$ if and only if $f-g \in \mathcal{A}_{s}^{+} P_{n}+P_{n}^{-}(\omega)$.

Obviously, the relations $\equiv, \equiv_{\omega}$ and $\simeq_{(s, \omega)}$ are equivalence ones. If $f \equiv_{\omega} g$, then $f \simeq{ }_{(s, \omega)} g$ for some $s \geqslant 0$. If $x$ is a monomial in $P_{n}$ and $\omega=\omega(x)$, then we denote $x \simeq_{s} g$ if and only if $x \simeq_{(s, \omega(x))} g$.

Proposition 2.1.4 (see [27]). Let $x, y$ be monomials and let $f, g$ be polynomials in $P_{n}$ such that $\operatorname{deg} x=\operatorname{deg} f, \operatorname{deg} y=\operatorname{deg} g$.

i) If $\omega_{i}(x) \leqslant 1$ for $i>s$ and $x \simeq_{t} f$ with $t \leqslant s$, then $x y^{2^{s}} \simeq_{t} f y^{2^{s}}$.

ii) If $\omega_{i}(x)=0$ for $i>s, x \simeq_{s} f$ and $y \simeq_{r} g$, then $x y^{2^{s}} \simeq_{s+r} f g^{2^{s}}$.

Denote by $Q P_{n}(\omega)$ the quotient of $P_{n}(\omega)$ by the equivalence relation $\equiv_{\omega}$. Then, we have

$$
Q P_{n}(\omega)=P_{n}(\omega) /\left(\left(\mathcal{A}^{+} P_{n} \cap P_{n}(\omega)\right)+P_{n}^{-}(\omega)\right) .
$$

It is easy to see that $\left(Q P_{n}\right)_{d} \cong \bigoplus_{\mathrm{deg} \omega=d} Q P_{n}(\omega)$.

We note that the weight vector of a monomial is invariant under the permutation of the generators $x_{i}$, hence $Q P_{n}(\omega)$ has an action of the symmetric group $\Sigma_{n}$. Furthermore, we have the following. 
Lemma 2.1.5 (see [29]). Let $\omega$ be a weight vector. Then, $Q P_{n}(\omega)$ is an $G L_{n^{-}}$ module.

For a polynomial $f \in P_{n}$, we denote by $[f]$ the class in $Q P_{n}$ represented by $f$. If $\omega$ is a weight vector, then denote by $[f]_{\omega}$ the class by the equivalence relation $\equiv_{\omega}$ which is represented by $f$. Denote by $|S|$ the cardinal of a set $S$.

For $1 \leqslant i \leqslant n$, define the $\mathcal{A}$-homomorphism $\rho_{i}: P_{n} \rightarrow P_{n}$, which is determined by $\rho_{i}\left(x_{i}\right)=x_{i+1}, \rho_{i}\left(x_{i+1}\right)=x_{i}, \rho_{i}\left(x_{j}\right)=x_{j}$ for $j \neq i, i+1,1 \leqslant i<n$, and $\rho_{n}\left(x_{1}\right)=x_{1}+x_{2}, \rho_{n}\left(x_{j}\right)=x_{j}$ for $j>1$.

It is easy to see that the general linear group $G L_{n}$ is generated by the matrices associated with $\rho_{i}, 1 \leqslant i \leqslant n$, and the symmetric group $\Sigma_{n}$ is generated by the ones associated with $\rho_{i}, 1 \leqslant i<n$. So, a class $[f]_{\omega}$ represented by a homogeneous polynomial $f \in P_{n}$ is an $G L_{n}$-invariant if and only if $\rho_{i}(f) \equiv_{\omega} f$ for $1 \leqslant i \leqslant n$. $[f]_{\omega}$ is an $\Sigma_{n}$-invariant if and only if $\rho_{i}(f) \equiv_{\omega} f$ for $1 \leqslant i<n$.

\subsection{The admissible monomial.}

Definition 2.2.1 (see Kameko [11]). Let $x, y$ be monomials of the same degree in $P_{n}$. We say that $x<y$ if and only if one of the following holds:

i) $\omega(x)<\omega(y)$;

ii) $\omega(x)=\omega(y)$ and $\sigma(x)<\sigma(y)$.

Definition 2.2.2 (see Kameko [1]). A monomial $x$ is said to be inadmissible if there exist monomials $y_{1}, y_{2}, \ldots, y_{m}$ such that $y_{t}<x$ for $t=1,2, \ldots, m$ and $x-\sum_{t=1}^{m} y_{t} \in \mathcal{A}^{+} P_{n}$.

A monomial $x$ is said to be admissible if it is not inadmissible.

Obviously, the set of all the admissible monomials of degree $d$ in $P_{n}$ is a minimal set of $\mathcal{A}$-generators for $P_{n}$ in degree $d$.

Theorem 2.2.3 (See Kameko [11], Sum [26]). Let $x, y, w$ be monomials in $P_{n}$ such that $\omega_{i}(x)=0$ for $i>r>0, \omega_{s}(w) \neq 0$ and $\omega_{i}(w)=0$ for $i>s>0$.

i) If $w$ is inadmissible, then $x w^{2^{r}}$ is also inadmissible.

ii) If $w$ is strictly inadmissible, then $w y^{2^{s}}$ is also strictly inadmissible.

Now, we recall a result of Singer [24] on the hit monomials in $P_{n}$.

Definition 2.2.4 (see Singer 24]). A monomial $z$ in $P_{n}$ is called a spike if $\nu_{j}(z)=$ $2^{s_{j}}-1$ for $s_{j}$ a non-negative integer and $j=1,2, \ldots, n$. If $z$ is a spike with $s_{1}>s_{2}>\ldots>s_{r-1} \geqslant s_{r}>0$ and $s_{j}=0$ for $j>r$, then it is called the minimal spike.

For a positive integer $d$, by $\mu(d)$ one means the smallest number $r$ for which it is possible to write $d=\sum_{1 \leqslant i \leqslant r}\left(2^{s_{i}}-1\right)$, where $s_{i}>0$. Singer showed in [24] that if $\mu(d) \leqslant n$, then there exists uniquely a minimal spike of degree $n$ in $P_{n}$.

Lemma 2.2.5 (See 18]). All the spikes in $P_{n}$ are admissible and their weight vectors are weakly decreasing. Furthermore, if a weight vector $\omega$ is weakly decreasing and $\omega_{1} \leqslant n$, then there is a spike $z$ in $P_{n}$ such that $\omega(z)=\omega$.

The following is a criterion for the hit monomials in $P_{n}$.

Theorem 2.2.6 (See Singer [24]). Suppose $x \in P_{n}$ is a monomial of degree $d$, where $\mu(d) \leqslant n$. Let $z$ be the minimal spike of degree $d$. If $\omega(x)<\omega(z)$, then $x$ is hit. 
From this theorem we see that if $z$ is the minimal spike of degree $d, \omega=\omega(z)$ and $f \in\left(P_{n}\right)_{d}$ then $[f]_{\omega}=[f]$. This result also implies a result of Wood, which originally is a conjecture of Peterson [17.

Theorem 2.2.7 (See Wood [36]). If $\mu(d)>n$, then $\left(Q P_{n}\right)_{d}=0$.

\subsection{Kameko's homomorphism.}

One of the main tools in the study of the hit problem is Kameko's homomorphism ${\widetilde{S q_{*}}}_{*}^{0}: Q P_{n} \rightarrow Q P_{n}$. This homomorphism is induced by the $\mathbb{F}_{2}$-linear map $\psi: P_{n} \rightarrow$ $P_{n}$, given by

$$
\psi(x)= \begin{cases}y, & \text { if } x=x_{1} x_{2} \ldots x_{n} y^{2}, \\ 0, & \text { otherwise }\end{cases}
$$

for any monomial $x \in P_{n}$. Note that $\psi$ is not an $\mathcal{A}$-homomorphism. However, $\psi S q^{2 i}=S q^{i} \psi$, and $\psi S q^{2 i+1}=0$ for any non-negative integer $i$.

Theorem 2.3.1 (Kameko [1]). Let $m$ be a positive integer. If $\mu(2 m+n)=n$, then ${\widetilde{S q_{*}}}_{*}^{0}=\left({\widetilde{S q_{*}}}^{0}\right)_{(n, m)}:\left(Q P_{n}\right)_{2 m+n} \rightarrow\left(Q P_{n}\right)_{m}$ is an isomorphism of the $G L_{n}$-modules.

We end this section by recalling some notations which will be used in the next sections. We set

$$
\begin{aligned}
& P_{n}^{0}=\left\langle\left\{x=x_{1}^{a_{1}} x_{2}^{a_{2}} \ldots x_{n}^{a_{n}}: a_{1} a_{2} \ldots a_{n}=0\right\}\right\rangle, \\
& P_{n}^{+}=\left\langle\left\{x=x_{1}^{a_{1}} x_{2}^{a_{2}} \ldots x_{n}^{a_{n}}: a_{1} a_{2} \ldots a_{n}>0\right\}\right\rangle .
\end{aligned}
$$

It is easy to see that $P_{n}^{0}$ and $P_{n}^{+}$are the $\mathcal{A}$-submodules of $P_{n}$. Furthermore, we have the following.

Proposition 2.3.2. We have a direct summand decomposition of the $\mathbb{F}_{2}$-vector spaces $Q P_{n}=Q P_{n}^{0} \oplus Q P_{n}^{+}$. Here $Q P_{n}^{0}=\mathbb{F}_{2} \otimes_{\mathcal{A}} P_{n}^{0}$ and $Q P_{n}^{+}=\mathbb{F}_{2} \otimes_{\mathcal{A}} P_{n}^{+}$.

\section{A construction for the generators of $P_{n}$}

We denote

$$
\mathcal{N}_{n}=\left\{(i ; I): I=\left(i_{1}, i_{2}, \ldots, i_{r}\right), 1 \leqslant i<i_{1}<\ldots<i_{r} \leqslant n, 0 \leqslant r<n\right\} .
$$

Let $(i ; I) \in \mathcal{N}_{n}$ and $j \in \mathbb{N}_{n}$. Denote by $r=\ell(I)$ the length of $I$, and

$$
I \cup j= \begin{cases}I, & \text { if } j \in I, \\ \left(i_{1}, \ldots, i_{t-1}, j, i_{t}, \ldots, i_{r}\right), & \text { if } i_{t-1}<j<i_{t}, 1 \leqslant t \leqslant r+1 .\end{cases}
$$

Here $i_{0}=0$ and $i_{r+1}=n+1$.

Definition 3.1 (see 27). Let $(i ; I) \in \mathcal{N}_{n}, r=\ell(I)$, and let $u$ be an integer with $1 \leqslant u \leqslant r$. A monomial $x \in P_{n-1}$ is said to be $u$-compatible with $(i ; I)$ if all of the following hold:

i) $\nu_{i_{1}-1}(x)=\nu_{i_{2}-1}(x)=\ldots=\nu_{i_{(u-1)}-1}(x)=2^{r}-1$,

ii) $\nu_{i_{u}-1}(x)>2^{r}-1$,

iii) $\alpha_{r-t}\left(\nu_{i_{u}-1}(x)\right)=1, \forall t, 1 \leqslant t \leqslant u$,

iv) $\alpha_{r-t}\left(\nu_{i_{t}-1}(x)\right)=1, \forall t, u<t \leqslant r$. 
Clearly, a monomial $x$ can be $u$-compatible with a given $(i ; I) \in \mathcal{N}_{n}$ for at most one value of $u$. By convention, $x$ is 1 -compatible with $(i ; \emptyset)$.

For $1 \leqslant i \leqslant n$, define the homomorphism $f_{i}: P_{n-1} \rightarrow P_{n}$ of algebras by substituting

$$
f_{i}\left(x_{j}\right)= \begin{cases}x_{j}, & \text { if } 1 \leqslant j<i, \\ x_{j+1}, & \text { if } i \leqslant j<n .\end{cases}
$$

Definition 3.2 (see [27]). Let $(i ; I) \in \mathcal{N}_{n}, x_{(I, u)}=x_{i_{u}}^{2^{r-1}+\ldots+2^{r-u}} \prod_{u<t \leqslant r} x_{i_{t}}^{2^{r-t}}$ for $r=\ell(I)>0, x_{(\emptyset, 1)}=1$. For a monomial $x$ in $P_{n-1}$, we define the monomial $\phi_{(i ; I)}(x)$ in $P_{n}$ by setting

$$
\phi_{(i ; I)}(x)= \begin{cases}\left(x_{i}^{2^{r}-1} f_{i}(x)\right) / x_{(I, u)}, & \text { if there exists } u \text { such that } \\ 0, & x \text { is } u \text {-compatible with }(i, I), \\ & \text { otherwise. }\end{cases}
$$

Then we have an $\mathbb{F}_{2}$-linear map $\phi_{(i ; I)}: P_{n-1} \rightarrow P_{n}$. In particular, $\phi_{(i ; \emptyset)}=f_{i}$.

It is easy to see that if $\phi_{(i ; I)}(x) \neq 0$ then $\omega\left(\phi_{(i ; I)}(x)\right)=\omega(x)$.

Definition 3.3. For any $(i ; I) \in \mathcal{N}_{n}$, we define the homomorphism $p_{(i ; I)}: P_{n} \rightarrow$ $P_{n-1}$ of algebras by substituting

$$
p_{(i ; I)}\left(x_{j}\right)= \begin{cases}x_{j}, & \text { if } 1 \leqslant j<i, \\ \sum_{s \in I} x_{s-1}, & \text { if } j=i, \\ x_{j-1}, & \text { if } i<j \leqslant n .\end{cases}
$$

Then, $p_{(i ; I)}$ is a homomorphism of $\mathcal{A}$-modules. In particular, for $I=\emptyset, p_{(i ; \emptyset)}\left(x_{i}\right)=0$ and $p_{(i ; I)}\left(f_{i}(y)\right)=y$ for any $y \in P_{n-1}$.

For a subset $B \subset P_{n}$, we denote $[B]=\{[f]: f \in B\}$. If $B \subset P_{n}(\omega)$, then we set $[B]_{\omega}=\left\{[f]_{\omega}: f \in B\right\}$.

We denote

$$
\begin{aligned}
\Phi^{0}(B) & =\bigcup_{1 \leqslant i \leqslant n} \phi_{(i ; \emptyset)}(B)=\bigcup_{1 \leqslant i \leqslant n} f_{i}(B) . \\
\Phi^{+}(B) & =\bigcup_{(i ; I) \in \mathcal{N}_{n}, 0<\ell(I) \leqslant n-1} \phi_{(i ; I)}(B) \backslash P_{n}^{0} . \\
\Phi(B) & =\Phi^{0}(B) \bigcup \Phi^{+}(B) .
\end{aligned}
$$

It is easy to see that if $B$ is a minimal set of generators for $\mathcal{A}$-module $P_{n-1}$ in degree $n$, then $\Phi^{0}(B)$ is a minimal set of generators for $\mathcal{A}$-module $P_{n}^{0}$ in degree $n$ and $\Phi^{+}(B) \subset P_{n}^{+}$.

Denote by $B_{n}(d)$ the set of all the admissible monomials of degree $d$ in $P_{n}$. Set $B_{n}^{0}(d)=B_{n}(d) \cap P_{n}^{0}, B_{n}^{+}(d)=B_{n}(d) \cap P_{n}^{+}$. If $\omega$ is a weight vector of degree $d$, then we set $B_{n}(\omega)=B_{n}(d) \cap P_{n}(\omega), B_{n}^{0}(\omega)=B_{n}(\omega) \cap P_{n}^{0}, B_{n}^{+}(\omega)=B_{n}(\omega) \cap P_{n}^{+}$.

The following gives our prediction on the relation between the admissible monomials for the polynomial algebras.

Conjecture 3.4. If $\omega$ is a weight vector, then $\Phi\left(B_{n-1}(\omega)\right) \subset B_{n}(\omega)$.

It is easy to see that if this conjecture is true, then $\Phi\left(B_{n-1}(d)\right) \subset B_{n}(d)$ for any positive integer $d$. 
From the results of Peterson [17, Kameko [11] and the present author [27, we can see that this conjecture is true for $n \leqslant 4$. In Section 5 we will show that this conjecture is true for $n=5$ and any weight vector $\omega$ of the degree $d=15.2^{s}-5$ for arbitrary non-negative integer $s$.

We recall some needed results in 27] which will be used later. For a positive integer $b$, denote

$$
\omega_{(n, b)}=\left((n-1)^{(b)}\right), \tilde{\omega}_{(n, b)}=\left((n-1)^{(b)}, 1\right) .
$$

Lemma 3.5 (See [27]). Let $b$ be a positive integer and let $j_{0}, j_{1}, \ldots, j_{b-1} \in \mathbb{N}_{n}$. We set $i=\min \left\{j_{0}, \ldots, j_{b-1}\right\}, I=\left(i_{1}, \ldots, i_{r}\right)$ with $\left\{i_{1}, \ldots, i_{r}\right\}=\left\{j_{0}, \ldots, j_{b-1}\right\} \backslash\{i\}$. Then, we have

$$
\prod_{0 \leqslant t<b} X_{j_{t}}^{2^{t}} \simeq_{b-1} \phi_{(i ; I)}\left(X^{2^{b}-1}\right) .
$$

Lemma 3.6 (See [27]). Let $i, j, d, a, b$ be positive integers such that $i, j \leqslant n, i \neq j$, and $a+b=2^{d}-1$. Then

$$
X_{i}^{a} X_{j}^{b} \simeq_{2} X_{i}^{2^{d}-2} X_{j} .
$$

Lemma 3.7 (See [27]). Let $(i ; I) \in \mathcal{N}_{n}$ and let $d, h, u$ be integers such that $\ell(I)=$ $r<h \leqslant d$, and $1 \leqslant i<u \leqslant n$. Then, we have

$$
\phi_{(i ; I)}\left(X^{2^{h}-1}\right) X_{u}^{2^{d}-2^{h}} \simeq_{r+2} \phi_{(i ; I \cup u)}\left(X^{2^{d}-1}\right) .
$$

We now prove some relations which are the usual tools for studying the hit problem.

Proposition 3.8. For any integer $0<\ell \leqslant n$,

$$
X_{\ell}^{2^{\ell}-1} x_{\ell}^{2^{\ell}} \simeq_{\ell} \sum_{u=\ell}^{n} \sum_{(i ; I) \in \mathcal{N}_{\ell-1}} \phi_{(i ; I \cup u)}\left(X^{2^{\ell}-1}\right) x_{u}^{2^{\ell}}+\sum_{u=\ell+1}^{n} X_{u}^{2^{\ell}-1} x_{u}^{2^{\ell}} .
$$

Proof. We prove the proposition by induction on $\ell$. For $\ell=1$,

$$
X_{1} x_{1}^{2}=\sum_{u=2}^{n} X_{u} x_{u}^{2}+S q^{1}\left(X_{\emptyset}\right) \simeq_{1} \sum_{u=2}^{n} X_{u} x_{u}^{2} .
$$

Since $\mathcal{N}_{0}=\emptyset$, the proposition is true.

Suppose that $1 \leqslant \ell<n$ and the proposition is true for $\ell$. Using the Cartan formula, we have

$$
\begin{aligned}
X_{\ell+1}^{2^{\ell+1}-1} x_{\ell+1}^{2^{\ell+1}} & =\sum_{u=1}^{\ell} X_{u}^{2^{\ell+1}-1} x_{u}^{2^{\ell+1}}+\sum_{u=\ell+2}^{n} X_{u}^{2^{\ell+1}-1} x_{u}^{2^{\ell+1}}+S q^{1}\left(X_{\emptyset}^{2^{\ell+1}-1}\right) \\
& \simeq_{1} \sum_{u=1}^{\ell} X_{u}^{2^{\ell+1-u}-1}\left(X_{u}^{2^{u}-1} x_{u}^{2^{u}}\right)^{2^{\ell+1-u}}+\sum_{u=\ell+2}^{n} X_{u}^{2^{\ell+1}-1} x_{u}^{2^{\ell+1}} .
\end{aligned}
$$

For $1 \leqslant u \leqslant \ell$, using the inductive hypothesis and Proposition 2.1.4 we get

$$
\begin{aligned}
X_{u}^{2^{\ell+1-u}-1}\left(X_{u}^{2^{u}-1} x_{u}^{2^{u}}\right)^{2^{\ell+1-u}} & \simeq_{\ell+1} X_{u}^{2^{\ell+1-u}-1}\left(\sum_{t=u+1}^{n} X_{t}^{2^{u}-1} x_{t}^{2^{u}}\right. \\
& \left.+\sum_{t=u}^{n} \sum_{(i ; I) \in \mathcal{N}_{u-1}} \phi_{(i ; I \cup t)}\left(X^{2^{u}-1}\right) x_{t}^{2^{u}}\right)^{2^{\ell+1-u}} .
\end{aligned}
$$


According to Lemma 3.5 and Proposition 2.1.4,

$$
\begin{aligned}
X_{u}^{2^{\ell+1-u}-1}\left(X_{t}^{2^{u}-1} x_{t}^{2^{u}}\right)^{2^{\ell+1-u}} & \simeq_{\ell} \phi_{(u ; t)}\left(X^{2^{\ell+1}-1}\right) x_{t}^{2^{\ell+1}}, \\
X_{u}^{2^{\ell+1-u}-1}\left(\phi_{(i ; I \cup t)}\left(X^{2^{u}-1}\right) x_{t}^{2^{u}}\right)^{2^{\ell+1-u}} & \simeq_{\ell} \phi_{(i ; I \cup t \cup u)}\left(X^{2^{\ell+1}-1}\right) x_{t}^{2^{\ell+1}} .
\end{aligned}
$$

From the above equalities, we get

$$
\begin{aligned}
X_{u}^{2^{\ell+1-u}-1}\left(X_{u}^{2^{u}-1} x_{u}^{2^{u}}\right)^{2^{\ell+1-u}} & \simeq_{\ell+1} \sum_{(i ; I) \in \mathcal{N}_{u-1}} \phi_{(i ; I \cup u)}\left(X^{2^{\ell+1}-1}\right) x_{u}^{2^{\ell+1}} \\
& +\sum_{t=u+1}^{n}\left(\sum_{(i ; I) \in \mathcal{N}_{u-1}} \phi_{(i ; I \cup t \cup u)}\left(X^{2^{\ell+1}-1}\right) x_{t}^{2^{\ell+1}}+\phi_{(u ; t)}\left(X^{2^{\ell+1}-1}\right) x_{t}^{2^{\ell+1}}\right) .
\end{aligned}
$$

For $2 \leqslant u \leqslant h \leqslant n$, we set $\mathcal{N}_{u-1} \cup u=\left\{(i ; I \cup u):(i ; I) \in \mathcal{N}_{u-1}\right\}$. Then we have

$$
\mathcal{N}_{h}=\left(\mathcal{N}_{1} \cup 2\right) \cup \ldots \cup\left(\mathcal{N}_{h-1} \cup h\right) \cup\{(1 ; \emptyset), \ldots,(h ; \emptyset)\} .
$$

By a direct computation from the above equalities and using the relation (3.1), we have

$$
\begin{aligned}
\sum_{u=1}^{\ell} X_{u}^{2^{\ell+1-u}-1}\left(X_{u}^{2^{u}-1} x_{u}^{2^{u}}\right)^{2^{\ell+1-u}} \simeq_{\ell+1} \sum_{u=2}^{\ell} \sum_{(i ; I) \in \mathcal{N}_{u-1}} \phi_{(i ; I \cup u)}\left(X^{2^{\ell+1}-1}\right) x_{u}^{2^{\ell+1}} \\
\quad+\sum_{t=2}^{\ell}\left(\sum_{u=1}^{t-1} \sum_{(i ; I) \in \mathcal{N}_{u-1} \cup u} \phi_{(i ; I \cup t)}\left(X^{2^{\ell+1}-1}\right)+\phi_{(u ; t)}\left(X^{2^{\ell+1}-1}\right)\right) x_{t}^{2^{\ell+1}} \\
\quad+\sum_{t=\ell+1}^{n}\left(\sum_{u=1}^{\ell} \sum_{(i ; I) \in \mathcal{N}_{u-1} \cup u} \phi_{(i ; I \cup t)}\left(X^{2^{\ell+1}-1}\right)+\phi_{(u ; t)}\left(X^{2^{\ell+1}-1}\right)\right) x_{t}^{2^{\ell+1}} \\
=\sum_{u=2}^{\ell} \sum_{(i ; I) \in \mathcal{N}_{u-1}} \phi_{(i ; I \cup u)}\left(X^{2^{\ell+1}-1}\right) x_{u}^{2^{\ell+1}}+\sum_{t=2}^{\ell} \sum_{t i ; I) \in \mathcal{N}_{t-1}} \phi_{(i ; I \cup t)}\left(X^{2^{\ell+1}-1}\right) x_{t}^{2^{\ell+1}} \\
=\sum_{t=\ell+1}^{n} \sum_{(i ; I \cup t) \in \mathcal{N}_{\ell}} \phi_{(i ; I \cup t)}\left(X^{2^{\ell+1}-1}\right) x_{t}^{2^{\ell+1}}
\end{aligned}
$$

Combining the above equalities, we get

$$
X_{\ell+1}^{2^{\ell+1}-1} x_{\ell+1}^{2^{\ell+1}} \simeq_{\ell+1} \sum_{u=\ell+1}^{n} \sum_{(i ; I) \in \mathcal{N}_{\ell}} \phi_{(i ; I \cup u)}\left(X^{2^{\ell+1}-1}\right) x_{u}^{2^{\ell+1}}+\sum_{u=\ell+2}^{n} X_{u}^{2^{\ell+1}-1} x_{u}^{2^{\ell+1}} .
$$

The proposition follows.

From the proof of this lemma, we obtain the following.

Corollary 3.9. For $2 \leqslant d \leqslant n$, we have

$$
\sum_{u=1}^{d-1} X_{u}^{2^{d}-1} x_{u}^{2^{d}} \simeq_{d} \sum_{u=d}^{n} \sum_{(i ; I) \in \mathcal{N}_{d-1}} \phi_{(i ; I \cup u)}\left(X^{2^{d}-1}\right) x_{u}^{2^{d}} .
$$


Proposition 3.10. Let $d, h, t, u$ be integers such that $1 \leqslant u<d-n+t, 0<t<$ $h \leqslant n$, and let $x$ be a monomial in $P_{n}$. Then we have

$$
Z:=\phi_{\left(t ; I_{t}\right)}\left(X^{2^{d-u}-1}\right) X_{h}^{2^{d}-2^{d-u}} x^{2^{d}} \simeq_{n-t+1} \phi_{\left(t ; I_{t}\right)}\left(X^{2^{d}-1}\right) x^{2^{d}} .
$$

Proof. We prove the proposition by double induction on $(t, u)$. If $t=n-1$, then $h=n$. By Lemma 3.6, we have

$$
\begin{aligned}
\phi_{(n-1 ; n)}\left(X^{2^{d-u}-1}\right) X_{n}^{2^{d}-2^{d-u}} x^{2^{d}} & =X_{n-1}^{2^{d-u}-2} X_{n}^{2^{d}-2^{d-u}+1} x^{2^{d}} \\
& \simeq_{2} \phi_{(n-1 ; n)}\left(X^{2^{d}-1}\right) x^{2^{d}} .
\end{aligned}
$$

So, the proposition holds. Suppose that $0<t<n-1$ and the proposition is true for $t+1$. If $h=t+1$, then

$Z=\phi_{\left(t+2 ; I_{t+2}\right)}\left(X^{2^{n-t-1}-1}\right)\left(X_{t}^{2^{d-n+t-u+1}-2} X_{t+1}^{2^{d-n+t+1}-2^{d-n+t-u+1}+1} x^{2^{d-n+t+1}}\right)^{2^{n-t-1}}$.

According to Lemma 3.6

$$
X_{t}^{2^{d-n+t-u+1}-2} X_{t+1}^{2^{d-n+t+1}-2^{d-n+t-u+1}+1} x^{2^{d-n+t+1}} \simeq_{2} X_{t}^{2^{d-n+t+1}-2} X_{t+1} x^{2^{d-n+t+1}} .
$$

Hence, using Proposition 2.1.4, we obtain

$$
\begin{aligned}
Z & \simeq_{n-t+1} \phi_{\left(t+2 ; I_{t+2}\right)}\left(X^{2^{n-t-1}-1}\right)\left(X_{t}^{2^{d-n+t+1}-2} X_{t+1} x^{2^{d-n+t+1}}\right)^{2^{n-t-1}} \\
& =\phi_{\left(t ; I_{t}\right)}\left(X^{2^{d}-1}\right) x^{2^{d}} .
\end{aligned}
$$

The proposition holds. Suppose that $h>t+1$ and $u=1$. We have

$$
Z=\phi_{\left(t+1 ; I_{t+1}\right)}\left(X^{2^{n-t}-1}\right)\left(X_{t}^{2^{d-n+t-1}-1} X_{h}^{2^{d-n+t-1}} x^{2^{d-n+t}}\right)^{2^{n-t}} .
$$

Since $X_{t}^{2^{d-n+t-1}-1} X_{h}^{2^{d-n+t-1}} x^{2^{d-n+t}} \simeq_{1} X_{t}^{2^{d-n+t-1}} X_{h}^{2^{d-n+t-1}-1} x^{2^{d-n+t}}$, applying Proposition 2.1.4 and the inductive hypothesis, we have

$$
\begin{aligned}
Z & \simeq_{n-t+1} \phi_{\left(t+1 ; I_{t+1}\right)}\left(X^{2^{n-t}-1}\right)\left(X_{t}^{2^{d-n+t-1}} X_{h}^{2^{d-n+t-1}-1} x^{2^{d-n+t}}\right)^{2^{n-t}} \\
& =\phi_{\left(t+1 ; I_{t+1}\right)}\left(X^{2^{n-t}-1}\right) X_{h}^{2^{d-1}-2^{n-t}}\left(X_{t} x^{2}\right)^{2^{d-1}} \\
& \simeq_{n-t} \phi_{\left(t+1 ; I_{t+1}\right)}\left(X^{2^{d-1}-1}\right)\left(X_{t} x^{2}\right)^{2^{d-1}} \\
& =\phi_{\left(t+2 ; I_{t+2}\right)}\left(X^{2^{n-t-1}-1}\right)\left(X_{t+1}^{2^{d-n+t}-1} X_{t}^{2^{d-n+t}} x^{2^{d-n+t+1}}\right)^{2^{n-t-1}} .
\end{aligned}
$$

According to Lemma 3.6

$$
X_{t+1}^{2^{d-n+t}-1} X_{t}^{2^{d-n+t}} x^{2^{d-n+t+1}} \simeq_{2} X_{t}^{2^{d-n+t+1}-2} X_{t+1} x^{2^{d-n+t+1}} .
$$

Hence, using Proposition 2.1.4, one gets

$$
\begin{aligned}
Z & \simeq_{n-t+1} \phi_{\left(t+2 ; I_{t+2}\right)}\left(X^{2^{n-t-1}-1}\right)\left(X_{t}^{2^{d-n+t+1}-2} X_{t+1} x^{2^{d-n+t+1}}\right)^{2^{n-t-1}} \\
& =\phi_{\left(t ; I_{t}\right)}\left(X^{2^{d}-1}\right) x^{2^{d}} .
\end{aligned}
$$

Now, suppose that $h>t+1$ and $u>1$. Set $d^{\prime}=d-u+1$. Combining Proposition 2.1.4 and the inductive hypothesis gives

$$
\begin{aligned}
Z & =\phi_{\left(t ; I_{t}\right)}\left(X^{2^{d^{\prime}-1}-1}\right) X_{h}^{2^{d^{\prime}}-2^{d^{\prime}-1}}\left(X_{h}^{2^{d-d^{\prime}}-1} x^{2^{d-d^{\prime}}}\right)^{2^{d^{\prime}}} \\
& \simeq_{n-t+1} \phi_{\left(t ; I_{t}\right)}\left(X^{2^{d^{\prime}}-1}\right)\left(X_{h}^{2^{d-d^{\prime}}-1} x^{2^{d-d^{\prime}}}\right)^{2^{d^{\prime}}} \\
& =\phi_{\left(t ; I_{t}\right)}\left(X^{2^{d-u+1}-1}\right) X_{h}^{2^{d}-2^{d-u+1}} x^{2^{d}} \simeq_{n-t+1} \phi_{\left(t ; I_{t}\right)}\left(X^{2^{d}-1}\right) x^{2^{d}} .
\end{aligned}
$$

The proposition is proved. 
Proposition 3.11. For any integer $d \geqslant n \geqslant 2$,

$$
X_{n}^{2^{d}-1} x_{n}^{2^{d}} \simeq_{n} \sum_{(i ; I) \in \mathcal{N}_{n-1}} \phi_{(i ; I \cup n)}\left(X^{2^{d}-1}\right) x_{n}^{2^{d}} .
$$

Proof. By Proposition 3.8, we have

$$
X_{n}^{2^{n}-1} x_{n}^{2^{n}} \simeq_{n} \sum_{(i ; I) \in \mathcal{N}_{n-1}} \phi_{(i ; I \cup n)}\left(X^{2^{n}-1}\right) x_{n}^{2^{n}} .
$$

Hence, using Proposition 2.1.4(i), we get

$$
X_{n}^{2^{d}-1} x_{n}^{2^{d}}=X_{n}^{2^{n}-1} x_{n}^{2^{n}} X_{\emptyset}^{2^{d}-2^{n}} \simeq_{n} \sum_{(i ; I) \in \mathcal{N}_{n-1}} \phi_{(i ; I \cup n)}\left(X^{2^{n}-1}\right) X_{n}^{2^{d}-2^{n}} x_{n}^{2^{d}} .
$$

Let $(i ; I) \in \mathcal{N}_{n-1}$. If $I=\emptyset$, then using Lemma 3.6, we have

$$
\begin{aligned}
& \phi_{(i ; I \cup n)}\left(X^{2^{n}-1}\right) X_{n}^{2^{d}-2^{n}} x_{n}^{2^{d}}=\phi_{(i ; n)}\left(X^{2^{n}-1}\right) X_{n}^{2^{d}-2^{n}} x_{n}^{2^{d}} \\
& =X_{i}^{2^{n}-2} X_{n}^{2^{d}-2^{n}+1} x_{n}^{2^{d}} \simeq_{2} X_{i}^{2^{d}-2} X_{n} x_{n}^{2^{d}}=\phi_{(i ; I \cup n)}\left(X^{2^{d}-1}\right) x_{n}^{2^{d}} .
\end{aligned}
$$

If $I=\left(i_{1}, \ldots, i_{r}\right), r>0$, then $s=n-\ell(I \cup n)>0$. Hence,

$$
\begin{aligned}
Y: & =\phi_{(i ; I \cup n)}\left(X^{2^{n}-1}\right) X_{n}^{2^{d}-2^{n}} x_{n}^{2^{d}} \\
& =\phi_{\left(i_{1} ; I \cup n \backslash i_{1}\right)}\left(X^{2^{n-s}-1}\right)\left(X_{i}^{2^{s}-1} X_{n}^{2^{d-n+s}-2^{s}} x_{n}^{2^{d-n+s}}\right)^{2^{n-s}} .
\end{aligned}
$$

By Lemma 3.6.

$$
X_{i}^{2^{s}-1} X_{n}^{2^{d-n+s}-2^{s}} x_{n}^{2^{d-n+s}} \simeq_{2} X_{i}^{2^{d-n+s}-2} X_{n} x_{n}^{2^{d-n+s}} .
$$

If $(i ; I \cup n)=\left(1 ; I_{1}\right)$, then using Proposition 3.10, we have

$$
\phi_{\left(1 ; I_{1}\right)}\left(X^{2^{n}-1}\right) X_{n}^{2^{d}-2^{n}} x_{n}^{2^{d}} \simeq_{n} \phi_{\left(1 ; I_{1}\right)}\left(X^{2^{d}-1}\right) x_{n}^{2^{d}} .
$$

If $(i ; I \cup n) \neq\left(1 ; I_{1}\right)$, then $s \geqslant 2$. Using Proposition 2.1.4 and Lemma 3.5, we obtain

$$
\begin{aligned}
Y & \simeq_{n-s+2} \phi_{\left(i_{1} ; I \cup n \backslash i_{1}\right)}\left(X^{2^{n-s}-1}\right)\left(X_{n} X_{i}^{2^{d-n+s}-2} x_{n}^{2^{d-n+s}}\right)^{2^{n-s}} \\
& =\left(\phi_{\left(i_{1} ; I \cup n \backslash i_{1}\right)}\left(X^{2^{n-s}-1}\right) X_{n}^{2^{n-s}} X_{i}^{2^{n-s+1}}\right) X_{i}^{2^{d}-2^{n-s+2}} x_{n}^{2^{d}} \\
& \simeq_{n-s+1} \phi_{(i ; I \cup n)}\left(X^{2^{n-s+2}-1}\right) X_{i}^{2^{d}-2^{n-s+2}} x_{n}^{2^{d}}=\phi_{(i ; I \cup n)}\left(X^{2^{d}-1}\right) x_{n}^{2^{d}} .
\end{aligned}
$$

Since $n-s+2 \leqslant n$, the proposition follows.

Now, using the above results we prove the following which has been proved in [27] by another method. Denote by $I_{t}=(t+1, t+2, \ldots, n)$ for $1 \leqslant t \leqslant n$. Set

$$
Y_{t}=Y_{n, t}=\sum_{u=t}^{n} \phi_{\left(t ; I_{t}\right)}\left(X^{2^{d}-1}\right) x_{u}^{2^{d}}, d>n-t+1 .
$$

Proposition 3.12 (See [27). For $1<t \leqslant n$,

$$
Y_{t} \simeq_{(n-t+1, \omega)} \sum_{(j ; J)} \phi_{(j ; J)}\left(X^{2^{d}-1}\right) x_{j}^{2^{d}},
$$

where the sum runs over some $(j ; J) \in \mathcal{N}_{n}$ with $1 \leqslant j<t, J \subset I_{t-1}, J \neq I_{t-1}$ and $\omega=\omega\left(X_{1}^{2^{d}-1} x_{1}^{2^{d}}\right)$. 
Lemma 3.13. For $d \geqslant n, Y_{1}=\sum_{u=1}^{n} \phi_{\left(1 ; I_{1}\right)}\left(X^{2^{d}-1}\right) x_{u}^{2^{d}} \simeq_{(n, \omega)} 0$, with $\omega=$ $\omega\left(X_{1}^{2^{d}-1} x_{1}^{2^{d}}\right)$. More precisely,

$$
Y_{1}=\sum_{0 \leqslant i<n} S q^{2^{i}}\left(y_{i}\right)+h
$$

with $y_{i}$ polynomials in $P_{n}$, and $h \in P_{n}^{-}(\omega)$.

Proof. First, we prove the following relation by induction on $t \geqslant 1$.

$$
Y_{1} \simeq_{(n, \omega)} Y_{t}+\sum_{u=t}^{n} \sum_{(i ; I) \in \mathcal{N}_{t-1}} \phi_{\left(i ; I \cup I_{t-1}\right)}\left(X^{2^{d}-1}\right) x_{u}^{2^{d}} .
$$

Since $\mathcal{N}_{0}=\emptyset$, the relation (3.2) is true for $t=1$. Note that if $1 \leqslant t<n$, then

$$
\phi_{\left(t ; I_{t}\right)}\left(X^{2^{d}-1}\right) x_{t}^{2^{d}}=\phi_{\left(t+1 ; I_{t+1}\right)}\left(X^{2^{n-t}-1}\right)\left(X_{t}^{2^{t}-1} x_{t}^{2^{t}} X_{\emptyset}^{2^{d-n+t}-2^{t}}\right)^{2^{n-t}} .
$$

Applying Proposition 3.8 and Proposition 2.1.4, we have

$$
\begin{aligned}
X_{t}^{2^{t}-1} x_{t}^{2^{t}} & X_{\emptyset}^{2^{d-n+t}-2^{t}} \simeq_{t} \sum_{u=t+1}^{n} X_{u}^{2^{d-n+t}-1} x_{u}^{2^{d-n+t}} \\
& +\sum_{u=t}^{n} \sum_{(i ; I) \in \mathcal{N}_{t-1}} \phi_{(i ; I \cup u)}\left(X^{2^{t}-1}\right) X_{u}^{2^{d-n+t}-2^{t}} x_{u}^{2^{d-n+t}} .
\end{aligned}
$$

Using Proposition 3.10, we obtain

$$
\phi_{\left(t+1 ; I_{t+1}\right)}\left(X^{2^{n-t}-1}\right) X_{u}^{2^{d}-2^{n-t}} x_{u}^{2^{d}} \simeq_{n-t} \phi_{\left(t+1 ; I_{t+1}\right)}\left(X^{2^{d}-1}\right) x_{u}^{2^{d}} .
$$

Applying Lemma 3.5 and Proposition 2.1.4 we have

$$
\begin{aligned}
\phi_{\left(t+1 ; I_{t+1}\right)}\left(X^{2^{n-t}-1}\right) & \left(\phi_{(i ; I \cup u)}\left(X^{2^{t}-1}\right)\right)^{2^{n-t}} X_{u}^{2^{d}-2^{n}} x_{u}^{2^{d}} \\
& \left.\simeq_{n-1} \phi_{\left(i ; I \cup I_{t} \cup u\right)}\left(X^{2^{n}-1}\right)\right) X_{u}^{2^{d}-2^{n}} x_{u}^{2^{d}}
\end{aligned}
$$

Combining the above equalities and Proposition 2.1.4 gives

$$
\phi_{\left(t ; I_{t}\right)}\left(X^{2^{d}-1}\right) x_{t}^{2^{d}} \simeq_{n} Y_{t+1}+\sum_{u=t}^{n} \sum_{(i ; I) \in \mathcal{N}_{t-1}} \phi_{\left(i ; I \cup I_{t} \cup u\right)}\left(X^{2^{n}-1}\right) X_{u}^{2^{d}-2^{n}} x_{u}^{2^{d}} .
$$

If $\left(i ; I \cup I_{t} \cup u\right)=\left(1 ; I_{1}\right)$, then by Proposition 3.10, we have

$$
\phi_{\left(1 ; I_{1}\right)}\left(X^{2^{n}-1}\right) X_{u}^{2^{d}-2^{n}} x_{u}^{2^{d}} \simeq_{n} \phi_{\left(1 ; I_{1}\right)}\left(X^{2^{d}-1}\right) x_{u}^{2^{d}} .
$$

Suppose $\left(i ; I \cup I_{t} \cup u\right) \neq\left(1 ; I_{1}\right)$. Then, $0<s=\ell\left(I \cup I_{t} \cup u\right) \leqslant n-2$. Using Lemma 3.7. we have

$$
\phi_{\left(1 ; I_{1}\right)}\left(X^{2^{n}-1}\right) X_{u}^{2^{d}-2^{n}} x_{u}^{2^{d}} \simeq_{s+2} \phi_{\left(i ; I \cup I_{t} \cup u\right)}\left(X^{2^{d}-1}\right) x_{u}^{2^{d}} .
$$

Combining the above equalities gives

$$
\phi_{\left(t ; I_{t}\right)}\left(X_{t}^{2^{d}-1}\right) x_{t}^{2^{d}} \simeq_{n} Y_{t+1}+\sum_{u=t}^{n} \sum_{(i ; I) \in \mathcal{N}_{t-1}} \phi_{\left(i ; I \cup I_{t} \cup u\right)}\left(X^{2^{d}-1}\right) x_{u}^{2^{d}} .
$$

Note that $I_{t} \cup t=I_{t-1}, I_{t} \cup u=I_{t}$ for $u>t$, and $\mathcal{N}_{t}=\mathcal{N}_{t-1} \cup\left(\mathcal{N}_{t-1} \cup t\right) \cup\{(t ; \emptyset)\}$. 
Computing from the last equalities and using the inductive hypothesis, we obtain

$$
\begin{aligned}
& Y_{1} \simeq_{(n, \omega)} Y_{t+1}+\sum_{u=t}^{n} \sum_{(i ; I) \in \mathcal{N}_{t-1}} \phi_{\left(i ; I \cup I_{t} \cup u\right)}\left(X^{2^{d}-1}\right) x_{u}^{2^{d}} \\
& \quad+\sum_{u=t+1}^{n} \phi_{\left(t ; I_{t}\right)}\left(X^{2^{d}-1}\right) x_{u}^{2^{d}}+\sum_{u=t}^{n} \sum_{(i ; I) \in \mathcal{N}_{t-1}} \phi_{\left(i ; I \cup I_{t-1}\right)}\left(X^{2^{d}-1}\right) x_{u}^{2^{d}} \\
&=Y_{t+1}+\sum_{u=t+1}^{n} \sum_{(i ; I) \in \mathcal{N}_{t-1}} \phi_{\left(i ; I \cup I_{t}\right)}\left(X^{2^{d}-1}\right) x_{u}^{2^{d}} \\
&+\sum_{u=t+1}^{n} \sum_{(i ; I) \in \mathcal{N}_{t-1} \cup t} \phi_{\left(i ; I \cup I_{t}\right)}\left(X^{2^{d}-1}\right) x_{u}^{2^{d}}+\sum_{u=t+1}^{n} \phi_{\left(t ; I_{t}\right)}\left(X^{2^{d}-1}\right) x_{u}^{2^{d}} \\
&= Y_{t+1}+\sum_{u=t+1}^{n} \sum_{(i ; I) \in \mathcal{N}_{t}} \phi_{\left(i ; I \cup I_{t}\right)}\left(X^{2^{d}-1}\right) x_{u}^{2^{d}} .
\end{aligned}
$$

The relation (3.2) is proved.

Since $Y_{n}=X_{n}^{2^{d}-1} x_{n}^{2^{d}}$, using the relation (3.2) with $t=n$ and Proposition 3.8 one gets

$$
Y_{1} \simeq_{(n, \omega)} X_{n}^{2^{d}-1} x_{n}^{2^{d}}+\sum_{(i ; I) \in \mathcal{N}_{n-1}} \phi_{(i ; I \cup n)}\left(X^{2^{d}-1}\right) x_{n}^{2^{d}} \simeq_{(n, \omega)} 0 .
$$

The lemma is proved.

Proof of Proposition 3.12. We have $Y_{t}=Z^{2^{d}-1} Y_{1}$ with $Z=x_{1} x_{2} \ldots x_{t-1}$ and $Y_{1}=$ $Y_{1}\left(x_{t}, \ldots, x_{n}\right) \in P_{n-t+1}:=\mathbb{F}_{2}\left[x_{t}, \ldots, x_{n}\right]$. Since $d \geqslant n-t+1$, by Lemma 3.13, $Y_{t}$ is a sum of polynomials of the form $f=Z^{2^{d}-1}\left(S q^{2^{i}}(y)+h\right)$ with $0 \leqslant i \leqslant n-t, y$ a monomial in $P_{n-t+1}$ and $h \in P_{n-t+1}^{-}\left(\omega^{*}\right)$ with $\omega^{*}=\omega\left(\left(x_{t+1} \ldots x_{n}\right)^{2^{d}-1} x_{t}^{2^{d}}\right)$.

Then $Z^{2^{d}-1} h \in P_{n}^{-}(\omega)$ with $\omega=\omega\left(X_{1}^{2^{d}-1} x_{1}^{2^{d}}\right)$. So, using the Cartan formula, we have

$$
f \simeq{ }_{(0, \omega)} S q^{2^{i}}\left(Z^{2^{d}-1} y\right)+\sum_{1 \leqslant v \leqslant 2^{i}} S q^{v}\left(Z^{2^{d}-1}\right) S q^{2^{i}-v}(y) .
$$

By a direct computation using the Cartan formula, we can easily show that if $1 \leqslant$ $v<2^{i}$, and a monomial $z$ appears as a term of the polynomial $S q^{v}\left(Z^{2^{d}-1}\right) S q^{2^{i}-v}(y)$, then $\omega_{u}(z)<n-1$ for some $u \leqslant d$. Hence, using the Cartan formula, one gets

$$
f \simeq_{(i+1, \omega)} S q^{2^{i}}\left(Z^{2^{d}-1}\right) y \simeq \simeq_{(0, \omega)} \sum_{0<j<t} Z^{2^{d}-1} x_{j}^{2^{i}} y .
$$

Since $\omega_{u}\left(Z^{2^{d}-1} x_{j}^{2^{i}}\right)=t-1$ for $u \leqslant i$ and $\omega_{u}\left(Z^{2^{d}-1} x_{j}^{2^{i}}\right)=t-2$ for $i<u \leqslant d$, if $Z^{2^{d}-1} x_{j}^{2^{i}} y \notin P_{n}^{-}(\omega)$, then $\omega_{u}(y)=n-t$ for $u \leqslant i$ and $\omega_{u}(y)=n-t+1$ for $i<u \leqslant d$. Hence, we obtain

$$
Z^{2^{d}-1} x_{j}^{2^{i}} y=\left(\prod_{0 \leqslant u \leqslant i-1} X_{j_{u}}^{2^{u}}\right) X_{j}^{2^{i}} X_{j}^{2^{d}-2^{i+1}} x_{j}^{2^{d}},
$$

where $j_{u} \geqslant t$, for $0 \leqslant u \leqslant i-1$. By convention, $\prod_{0 \leqslant u \leqslant i-1} X_{j_{u}}^{2^{u}}=1$ for $i=0$. 
According to Lemma 3.5 there is $J \subset\left\{j_{0}, \ldots, j_{i-1}\right\} \subset I_{t-1}$ such that

$$
\left(\prod_{0 \leqslant u \leqslant i-1} X_{j_{u}}^{2^{u}}\right) X_{j}^{2^{i}} \simeq_{i} \phi_{(j ; J)}\left(X^{2^{i+1}-1}\right) .
$$

From the above equalities and Proposition 2.1.4, we get

$$
Z^{2^{d}-1} x_{j}^{2^{i}} y \simeq_{(i+1, \omega)} \phi_{(j ; J)}\left(X^{2^{i+1}-1}\right) X_{j}^{2^{d}-2^{i+1}} x_{j}^{2^{d}}=\phi_{(j ; J)}\left(X^{2^{d}-1}\right) x_{j}^{2^{d}} .
$$

We have $\ell(J) \leqslant i \leqslant n-t<n-t+1=\ell\left(I_{t-1}\right)$. Hence, $J \neq I_{t-1}$. Since $i+1 \leqslant n-t+1$, the proposition follows.

\section{The admissible monomials of the Degree $d=15.2^{s}-5$ in $P_{5}$}

By using Theorem 2.2.7 we can show that the hit problem is reduced to the case of the degree of the form $d=t\left(2^{s}-1\right)+2^{s} m$ with $t, s, m$ positive integers such that $\mu(m)<t \leqslant n$ (see [27]). For $t=n$, the problem has been studied by Hưng [8] and by Tín and Sum [34. For $t=n=5$, it is explicitly computed by Tín [31, 32, 33] with $m=1,2,3$ and by the present author 29] with $m=5$. These results show that Conjecture 3.4 is true in those cases.

In this section we study this problem for $n=t=5$ and $m=10$. More precisely, we explicitly determine the admissible monomials of degree $d=15.2^{s}-5$ in $P_{5}$.

It is easy to see that for $s>1$, we have $\mu\left(15.2^{s}-5\right)=5$. Hence, Theorem 2.3.1 implies that

$$
\left(\widetilde{S q}_{*}^{0}\right)^{s-1}:\left(Q P_{5}\right)_{15.2^{s-5}} \longrightarrow\left(Q P_{5}\right)_{25}
$$

is an isomorphism of $G L_{5}$-modules for every $s \geqslant 1$. So, we need only to compute the space $\left(Q P_{5}\right)_{15.2^{s}-5}$ for $s=1$. Since Kameko's homomorphism

$$
\left(\widetilde{S q}_{*}^{0}\right)_{(5,10)}:\left(Q P_{5}\right)_{25} \longrightarrow\left(Q P_{5}\right)_{10}
$$

is an epimorphism, we have $\left(Q P_{5}\right)_{25} \cong \operatorname{Ker}\left(\widetilde{S q}_{*}^{0}\right)_{(5,10)} \bigoplus\left(Q P_{5}\right)_{10}$.

The admissible monomials of the degree 10 in $P_{5}$ have been determined by Tín [33. We have $B_{5}(10)=\left\{a_{10, t}: 1 \leqslant t \leqslant 280\right\}$, where the monomials $a_{10, t}, 1 \leqslant t \leqslant$ 280, are listed in Subsection 6.1. We now compute $\operatorname{Ker}\left(\widetilde{S q}_{*}^{0}\right)_{(5,10)}$.

Lemma 4.1. If $x$ is an admissible monomial of degree 25 in $P_{5}$ and $[x]$ belongs to $\operatorname{Ker}\left(\widetilde{S q}_{*}^{0}\right)_{(5,10)}$, then either $\omega(x)=(3,3,2,1)$ or $\omega(x)=(3,3,4)$.

Proof. Note that $z=x_{1}^{15} x_{2}^{7} x_{3}^{3}$ is the minimal spike of degree 25 in $P_{5}$ and $\omega(z)=$ $(3,3,2,1)$. Since $[x] \neq 0$, by Theorem 2.2.6 either $\omega_{1}(x)=3$ or $\omega_{1}(x)=5$. If $\omega_{1}(x)=5$, then $x=X_{\emptyset} y^{2}$ with $y$ a monomial of degree 10 in $P_{5}$. Since $x$ is admissible, by Theorem 2.2.3, $y$ is also admissible. Hence, $\left(\widetilde{S q}_{*}^{0}\right)_{(5,10)}([x])=[y] \neq 0$. This contradicts the fact that $[x] \in \operatorname{Ker}\left(\widetilde{S q}_{*}^{0}\right)_{(5,10)}$, hence $\omega_{1}(x)=3$. Then, we have $x=x_{i} x_{j} x_{\ell} y_{1}^{2}$ with $1 \leqslant i<j<\ell \leqslant 5$ and $y_{1}$ an admissible monomial of degree 11 in $P_{5}$. According to Tín [31, either $\omega\left(y_{1}\right)=(3,2,1)$ or $\omega\left(y_{1}\right)=(3,4)$. The lemma follows.

By Lemma 4.1 we have $\operatorname{Ker}\left(\widetilde{S q}_{*}^{0}\right)_{(5,10)} \cong Q P_{5}(3,3,2,1) \oplus Q P_{5}(3,3,4)$.

Proposition 4.2. $Q P_{5}(3,3,4)=0$.

To prove this proposition, we need some lemmas. By a simple computation one gets the two following lemmas. 
Lemma 4.3. If $x$ is one of the following monomials, then $f_{i}(x), 1 \leqslant i \leqslant 5$, are strictly inadmissible:

$$
x_{1}^{2} x_{2} x_{3}^{3} x_{4}^{3} \quad x_{1}^{2} x_{2}^{3} x_{3} x_{4}^{3} \quad x_{1}^{2} x_{2}^{3} x_{3}^{3} x_{4} \quad x_{1}^{3} x_{2}^{2} x_{3} x_{4}^{3} \quad x_{1}^{3} x_{2}^{2} x_{3}^{3} x_{4} \quad x_{1}^{3} x_{2}^{3} x_{3}^{2} x_{4}
$$

Lemma 4.4. The following monomials are strictly inadmissible:

$$
\begin{array}{lllll}
x_{1}^{2} x_{2} x_{3} x_{4}^{2} x_{5}^{3} & x_{1}^{2} x_{2} x_{3} x_{4}^{3} x_{5}^{2} & x_{1}^{2} x_{2} x_{3}^{2} x_{4} x_{5}^{3} & x_{1}^{2} x_{2} x_{3}^{2} x_{4}^{3} x_{5} & x_{1}^{2} x_{2} x_{3}^{3} x_{4} x_{5}^{2} \\
x_{1}^{2} x_{2} x_{3}^{3} x_{4}^{2} x_{5} & x_{1}^{2} x_{2}^{2} x_{3} x_{4} x_{5}^{3} & x_{1}^{2} x_{2}^{2} x_{3} x_{4}^{3} x_{5} & x_{1}^{2} x_{2}^{2} x_{3}^{3} x_{4} x_{5} & x_{1}^{2} x_{2}^{3} x_{3} x_{4} x_{5}^{2} \\
x_{1}^{2} x_{2}^{3} x_{3} x_{4}^{2} x_{5} & x_{1}^{2} x_{2}^{3} x_{3}^{2} x_{4} x_{5} & x_{1}^{3} x_{2}^{2} x_{3} x_{4} x_{5}^{2} & x_{1}^{3} x_{2}^{2} x_{3} x_{4}^{2} x_{5} & x_{1}^{3} x_{2}^{2} x_{3}^{2} x_{4} x_{5}
\end{array}
$$

Lemma 4.5. All permutations of the following monomials are strictly inadmissible:

$$
x_{1}^{3} x_{2}^{4} x_{3}^{4} x_{4}^{7} x_{5}^{7} \quad x_{1}^{3} x_{2}^{4} x_{3}^{5} x_{4}^{6} x_{5}^{7} \quad x_{1}^{3} x_{2}^{5} x_{3}^{5} x_{4}^{6} x_{5}^{6}
$$

Proof. We prove the lemma for the monomial $x=x_{1}^{3} x_{2}^{4} x_{3}^{4} x_{4}^{7} x_{5}^{7}$. The others can be proved by the similar computations. By a direct computation, we have

$$
x=S q^{1}\left(x_{1}^{5} x_{2} x_{3}^{4} x_{4}^{7} x_{5}^{7}\right)+S q^{2}\left(x_{1}^{3} x_{2}^{2} x_{3}^{4} x_{4}^{7} x_{5}^{7}+x_{1}^{6} x_{2} x_{3}^{2} x_{4}^{7} x_{5}^{7}\right) \bmod \left(P_{5}^{-}(3,3,4)\right) .
$$

This equality shows that all permutations of $x$ are strictly inadmissible.

Proof of Proposition 4.2. Let $x$ be an admissible monomial in $P_{5}$ such that $\omega(x)=$ $(3,3,4)$. Then $x=x_{j} x_{\ell} x_{t} y^{2}$ with $y \in B_{5}(3,4)$ and $1 \leqslant j<\ell<t \leqslant 5$. Let $z \in B_{5}(3,4)$ such that $x_{j} x_{\ell} x_{t} z^{2} \in P_{5}^{+}$. By a direct computation we see that if $x_{j} x_{\ell} x_{t} z^{2}$ is not a permutation of one of monomials as given in Lemma4.5, then there is a monomial $w$ which is given in one of Lemmas 4.3, 4.4 such that $x_{j} x_{\ell} x_{t} z^{2}=w z_{1}^{2^{u}}$ with suitable monomial $z_{1} \in P_{5}$, and $u=\max \left\{j \in \mathbb{Z}: \omega_{j}(w)>0\right\}$. By Theorem 2.2.3. $x_{j} x_{\ell} x_{t} z^{2}$ is inadmissible. Since $x=x_{j} x_{\ell} x_{t} y^{2}$ and $x$ is admissible, $x$ is a permutation of one of monomials as given in Lemma 4.5. Now the proposition follows from Lemma 4.5 .

By computing from a result in [27, we have

$$
B_{5}^{0}(25)=B_{5}^{0}(3,3,2,1)=\left\{a_{25, t}: 1 \leqslant t \leqslant 520\right\},
$$

where the monomials $a_{25, t}, 1 \leqslant t \leqslant 520$, are listed in Subsection 6.3

Proposition 4.6. There exist exactly 440 admissible monomials in $P_{5}^{+}$such that their weight vectors are $(3,3,2,1)$. Consequently $\operatorname{dim} Q P_{5}^{+}(3,3,2,1)=440$.

We prove the proposition by showing that $B_{5}^{+}(3,3,2,1)=\left\{b_{25, u}: 1 \leqslant u \leqslant 440\right\}$, where the monomials $b_{25, u}, 1 \leqslant u \leqslant 440$, are listed in Subsection 6.4

We need some lemmas for the proof of this proposition. The following is a corollary of a result in [27].

Lemma 4.7. If $x$ is one of the following monomials then $f_{i}(x), 1 \leqslant i \leqslant 5$, are strictly inadmissible:

$$
\begin{array}{llll}
x_{1}^{3} x_{2}^{4} x_{3}^{3} x_{4}^{7} & x_{1}^{7} x_{2}^{9} x_{3}^{2} x_{4}^{7} & x_{1}^{7} x_{2}^{9} x_{3}^{7} x_{4}^{2} & x_{1}^{7} x_{2}^{9} x_{3}^{6} x_{4}^{3} \\
x_{1}^{3} x_{2}^{7} x_{3}^{8} x_{4}^{7} & x_{1}^{7} x_{2}^{3} x_{3}^{8} x_{4}^{7} & x_{1}^{7} x_{2}^{8} x_{3}^{3} x_{4}^{7} & x_{1}^{7} x_{2}^{8} x_{3}^{7} x_{4}^{3}
\end{array}
$$

Lemma 4.8. The following monomials are strictly inadmissible:

$$
\begin{array}{lllll}
x_{1} x_{2}^{2} x_{3}^{6} x_{4} x_{5}^{7} & x_{1} x_{2}^{2} x_{3}^{6} x_{4}^{7} x_{5} & x_{1} x_{2}^{2} x_{3}^{7} x_{4}^{6} x_{5} & x_{1} x_{2}^{6} x_{3}^{2} x_{4} x_{5}^{7} & x_{1} x_{2}^{6} x_{3}^{2} x_{4}^{7} x_{5} \\
x_{1} x_{2}^{6} x_{3}^{7} x_{4}^{2} x_{5} & x_{1} x_{2}^{7} x_{3}^{2} x_{4}^{6} x_{5} & x_{1} x_{2}^{7} x_{3}^{6} x_{4}^{2} x_{5} & x_{1}^{7} x_{2} x_{3}^{2} x_{4}^{6} x_{5} & x_{1}^{7} x_{2} x_{3}^{6} x_{4}^{2} x_{5} \\
x_{1} x_{2}^{6} x_{3}^{3} x_{4}^{6} x_{5} & x_{1} x_{2}^{6} x_{3}^{6} x_{4} x_{5}^{3} & x_{1} x_{2}^{6} x_{3}^{6} x_{4}^{3} x_{5} & x_{1} x_{2}^{2} x_{3}^{2} x_{4}^{5} x_{5}^{7} & x_{1} x_{2}^{2} x_{3}^{2} x_{4}^{7} x_{5}^{5} \\
x_{1} x_{2}^{2} x_{3}^{7} x_{4}^{2} x_{5}^{5} & x_{1} x_{2}^{7} x_{3}^{2} x_{4}^{2} x_{5}^{5} & x_{1}^{7} x_{2} x_{3}^{2} x_{4}^{2} x_{5}^{5} & x_{1} x_{2}^{2} x_{3}^{6} x_{4}^{3} x_{5}^{5} & x_{1} x_{2}^{2} x_{3}^{6} x_{4}^{5} x_{5}^{3} \\
x_{1} x_{2}^{6} x_{3}^{2} x_{4}^{3} x_{5}^{5} & x_{1} x_{2}^{6} x_{3}^{2} x_{4}^{5} x_{5}^{3} & x_{1} x_{2}^{6} x_{3}^{3} x_{4}^{2} x_{5}^{5} & x_{1} x_{2}^{6} x_{3}^{3} x_{4}^{4} x_{5}^{3} & x_{1}^{3} x_{2}^{4} x_{3} x_{4}^{6} x_{5}^{3} \\
x_{1}^{3} x_{2}^{4} x_{3}^{3} x_{4}^{3} x_{5}^{4} & x_{1}^{3} x_{2}^{4} x_{3}^{3} x_{4}^{4} x_{5}^{3} & & &
\end{array}
$$


Proof. We prove this lemma for the monomial $x=x_{1} x_{2}^{2} x_{3}^{6} x_{4}^{7} x_{5}$. The others can be proved by the similar computations. We have

$$
\begin{aligned}
x= & x_{1} x_{2}^{2} x_{3}^{5} x_{4}^{7} x_{5}^{2}+x_{1} x_{2} x_{3}^{6} x_{4}^{7} x_{5}^{2}+S q^{1}\left(x_{1}^{2} x_{2} x_{3}^{5} x_{4}^{7} x_{5}+x_{1}^{2} x_{2} x_{3}^{3} x_{4}^{9} x_{5}\right) \\
& +S q^{2}\left(x_{1} x_{2} x_{3}^{5} x_{4}^{7} x_{5}+x_{1} x_{2} x_{3}^{3} x_{4}^{9} x_{5}\right) \bmod \left(P_{5}^{-}(3,3,2,1)\right) .
\end{aligned}
$$

This equality shows that $x$ is strictly inadmissible.

Lemma 4.9. The following monomials are strictly inadmissible:

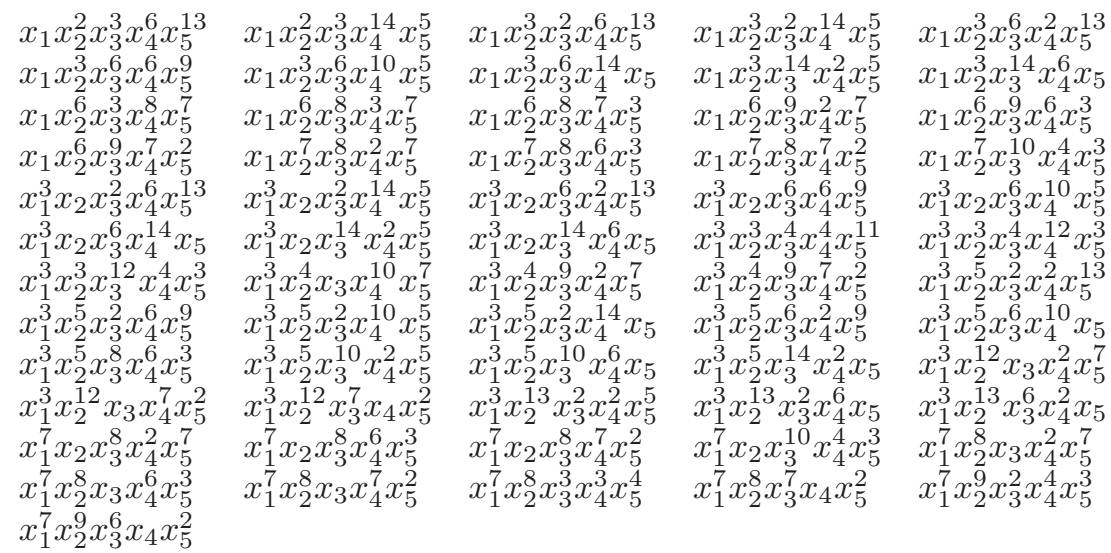

Proof. We prove this lemma for the monomial $x=x_{1}^{3} x_{2}^{5} x_{3}^{8} x_{4}^{6} x_{5}^{3}$. The others can be proved by the similar computations. We have

$$
\begin{aligned}
& x=x_{1}^{2} x_{2}^{3} x_{3}^{5} x_{4}^{6} x_{5}^{9}+x_{1}^{2} x_{2}^{3} x_{3}^{5} x_{4}^{9} x_{5}^{6}+x_{1}^{2} x_{2}^{3} x_{3}^{6} x_{4}^{5} x_{5}^{9}+x_{1}^{2} x_{2}^{3} x_{3}^{6} x_{4}^{9} x_{5}^{5}+x_{1}^{2} x_{2}^{3} x_{3}^{9} x_{4}^{5} x_{5}^{6} \\
& +x_{1}^{2} x_{2}^{3} x_{3}^{9} x_{4}^{6} x_{5}^{5}+x_{1}^{2} x_{2}^{5} x_{3}^{3} x_{4}^{6} x_{5}^{9}+x_{1}^{2} x_{2}^{5} x_{3}^{3} x_{4}^{9} x_{5}^{6}+x_{1}^{2} x_{2}^{5} x_{3}^{6} x_{4}^{3} x_{5}^{9}+x_{1}^{2} x_{2}^{5} x_{3}^{6} x_{4}^{9} x_{5}^{3} \\
& +x_{1}^{2} x_{2}^{5} x_{3}^{9} x_{4}^{3} x_{5}^{6}+x_{1}^{2} x_{2}^{5} x_{3}^{9} x_{4}^{6} x_{5}^{3}+x_{1}^{3} x_{2}^{3} x_{3}^{4} x_{4}^{6} x_{5}^{9}+x_{1}^{3} x_{2}^{3} x_{3}^{4} x_{4}^{9} x_{5}^{6}+x_{1}^{3} x_{2}^{3} x_{3}^{5} x_{4}^{6} x_{5}^{8} \\
& +x_{1}^{3} x_{2}^{3} x_{3}^{5} x_{4}^{8} x_{5}^{6}+x_{1}^{3} x_{2}^{3} x_{3}^{6} x_{4}^{4} x_{5}^{9}+x_{1}^{3} x_{2}^{3} x_{3}^{6} x_{4}^{5} x_{5}^{8}+x_{1}^{3} x_{2}^{3} x_{3}^{6} x_{4}^{8} x_{5}^{5}+x_{1}^{3} x_{2}^{3} x_{3}^{6} x_{4}^{9} x_{5}^{4} \\
& +x_{1}^{3} x_{2}^{3} x_{3}^{8} x_{4}^{5} x_{5}^{6}+x_{1}^{3} x_{2}^{3} x_{3}^{8} x_{4}^{6} x_{5}^{5}+x_{1}^{3} x_{2}^{3} x_{3}^{9} x_{4}^{4} x_{5}^{6}+x_{1}^{3} x_{2}^{3} x_{3}^{9} x_{4}^{6} x_{5}^{4}+x_{1}^{3} x_{2}^{4} x_{3}^{3} x_{4}^{6} x_{5}^{9} \\
& +x_{1}^{3} x_{2}^{4} x_{3}^{3} x_{4}^{9} x_{5}^{6}+x_{1}^{3} x_{2}^{4} x_{3}^{6} x_{4}^{3} x_{5}^{9}+x_{1}^{3} x_{2}^{4} x_{3}^{6} x_{4}^{9} x_{5}^{3}+x_{1}^{3} x_{2}^{4} x_{3}^{9} x_{4}^{3} x_{5}^{6}+x_{1}^{3} x_{2}^{4} x_{3}^{9} x_{4}^{6} x_{5}^{3} \\
& +x_{1}^{3} x_{2}^{5} x_{3}^{3} x_{4}^{6} x_{5}^{8}+x_{1}^{3} x_{2}^{5} x_{3}^{3} x_{4}^{8} x_{5}^{6}+x_{1}^{3} x_{2}^{5} x_{3}^{6} x_{4}^{3} x_{5}^{8}+x_{1}^{3} x_{2}^{5} x_{3}^{6} x_{4}^{8} x_{5}^{3}+x_{1}^{3} x_{2}^{5} x_{3}^{8} x_{4}^{3} x_{5}^{6} \\
& +S q^{1}\left(x_{1}^{3} x_{2}^{3} x_{3}^{3} x_{4}^{6} x_{5}^{9}+x_{1}^{3} x_{2}^{3} x_{3}^{3} x_{4}^{9} x_{5}^{6}+x_{1}^{3} x_{2}^{3} x_{3}^{6} x_{4}^{3} x_{5}^{9}+x_{1}^{3} x_{2}^{3} x_{3}^{6} x_{4}^{9} x_{5}^{3}+x_{1}^{3} x_{2}^{3} x_{3}^{9} x_{4}^{3} x_{5}^{6}\right. \\
& \left.+x_{1}^{3} x_{2}^{3} x_{3}^{9} x_{4}^{6} x_{5}^{3}+x_{1}^{3} x_{2}^{6} x_{3}^{5} x_{4}^{5} x_{5}^{5}\right)+S q^{2}\left(x_{1}^{2} x_{2}^{3} x_{3}^{3} x_{4}^{6} x_{5}^{9}+x_{1}^{2} x_{2}^{3} x_{3}^{3} x_{4}^{9} x_{5}^{6}+x_{1}^{2} x_{2}^{3} x_{3}^{6} x_{4}^{3} x_{5}^{9}\right. \\
& +x_{1}^{2} x_{2}^{3} x_{3}^{6} x_{4}^{9} x_{5}^{3}+x_{1}^{2} x_{2}^{3} x_{3}^{9} x_{4}^{3} x_{5}^{6}+x_{1}^{2} x_{2}^{3} x_{3}^{9} x_{4}^{6} x_{5}^{3}+x_{1}^{3} x_{2}^{5} x_{3}^{5} x_{4}^{5} x_{5}^{5}+x_{1}^{3} x_{2}^{5} x_{3}^{5} x_{4}^{5} x_{5}^{5} \\
& +x_{1}^{5} x_{2}^{3} x_{3}^{3} x_{4}^{6} x_{5}^{6}+x_{1}^{5} x_{2}^{3} x_{3}^{5} x_{4}^{5} x_{5}^{5}+x_{1}^{5} x_{2}^{3} x_{3}^{6} x_{4}^{3} x_{5}^{6}+x_{1}^{5} x_{2}^{3} x_{3}^{6} x_{4}^{6} x_{5}^{3}+x_{1}^{5} x_{2}^{5} x_{3}^{3} x_{4}^{5} x_{5}^{5} \\
& \left.+x_{1}^{5} x_{2}^{5} x_{3}^{5} x_{4}^{3} x_{5}^{5}\right)+S q^{4}\left(x_{1}^{3} x_{2}^{3} x_{3}^{3} x_{4}^{3} x_{5}^{9}+x_{1}^{3} x_{2}^{3} x_{3}^{3} x_{4}^{6} x_{5}^{6}+x_{1}^{3} x_{2}^{3} x_{3}^{6} x_{4}^{3} x_{5}^{6}\right. \\
& \left.+x_{1}^{3} x_{2}^{3} x_{3}^{6} x_{4}^{6} x_{5}^{3}\right)+S q^{8}\left(x_{1}^{3} x_{2}^{3} x_{3}^{3} x_{4}^{3} x_{5}^{5}\right) \bmod \left(P_{5}^{-}(3,3,2,1)\right) .
\end{aligned}
$$

This equality implies that $x$ is strictly inadmissible.

Proof of Proposition 4.6. Let $x$ be an admissible monomial in $\left(P_{5}^{+}\right)_{25}$ such that $\omega(x)=(3,3,2,1)$. Then, $x=x_{j} x_{\ell} x_{t} y^{2}$ with $1 \leqslant j<\ell<t \leqslant 5$ and $y \in B_{5}(3,2,1)$.

Let $z \in B_{5}(3,2,1)$ such that $x_{j} x_{\ell} x_{t} z^{2} \in P_{5}^{+}$. By a direct computation using the results in Tín [31, we see that if $x_{j} x_{\ell} x_{t} z^{2} \neq b_{25, u}, \forall u, 1 \leqslant u \leqslant 440$, then there is a monomial $w$ which is given in one of Lemmas 4.3 4.7. 4.8 and 4.9 such that 
$x_{j} x_{\ell} x_{t} z^{2}=w z_{1}^{2^{r}}$ with suitable monomial $z_{1} \in P_{5}$, and $r=\max \left\{j \in \mathbb{Z}: \omega_{j}(w)>0\right\}$. By Theorem 2.2.3, $x_{j} x_{\ell} x_{t} z^{2}$ is inadmissible. Since $x$ is admissible and $x=x_{j} x_{\ell} x_{t} y^{2}$ with $y \in B_{5}(3,2,1)$, one gets $x=b_{25, u}$ for some $u, 1 \leqslant u \leqslant 440$. This implies $B_{5}^{+}(3,3,2,1) \subset\left\{b_{25, u}: 1 \leqslant u \leqslant 440\right\}$.

We now prove the set $\left\{\left[b_{25, u}\right]: 1 \leqslant u \leqslant 440\right\}$ is linearly independent in the space $\left(\mathbb{F}_{2} \otimes_{\mathcal{A}} P_{5}\right)_{25}$. Suppose there is a linear relation

$$
\mathcal{S}=\sum_{u=1}^{440} \gamma_{u} b_{25, u} \equiv 0,
$$

where $\gamma_{u} \in \mathbb{F}_{2}$. For $1 \leqslant i<j \leqslant 5$, we explicitly compute $p_{(i ; I)}(\mathcal{S})$ in terms of the admissible monomials in $P_{4}\left(\bmod \left(\mathcal{A}^{+} P_{4}\right)\right)$. By a direct computation from the relations $p_{(i ; I)}(\mathcal{S}) \equiv 0$ with $\ell(I) \leqslant 2$, we obtain $\gamma_{u}=0$ for $1 \leqslant u \leqslant 440$. The proposition follows.

We have $\operatorname{dim}\left(Q P_{5}\right)_{10}=280$ and $\operatorname{dim}\left(Q P_{5}^{0}\right)_{25}=520$. So, one gets the following theorem.

Theorem 4.10. For any positive integer $s$, we have $\operatorname{dim}\left(Q P_{5}\right)_{15.2^{s}-5}=1240$.

\section{An application to the Fifth Singer transfer}

In this section, we verify Singer's conjecture for the fifth algebraic tranfer in the internal degree $15.2^{s}-5$ by using the results in Section 4 . The main result of the section is the following.

Theorem 5.1. For any non-negative integer $s$, we have $\left(Q P_{5}\right)_{15.2^{s}-5}^{G L_{5}}=0$.

By using the results of Tangora [30, Lin [12] and Chen [5], we easily obtain $\operatorname{Tor}_{5,7.2^{s}}^{\mathcal{A}}\left(\mathbb{F}_{2}, \mathbb{F}_{2}\right)=0$. So, by Theorem 5.1 the homomorphism

$$
\varphi_{5}: \operatorname{Tor}_{5,15.2^{s}}^{\mathcal{A}}\left(\mathbb{F}_{2}, \mathbb{F}_{2}\right) \longrightarrow\left(\mathbb{F}_{2} \otimes_{\mathcal{A}} P_{5}\right)_{15.2^{s}-5}^{G L_{5}}
$$

is a trivial isomorphism. Hence, one gets the following.

Corollary 5.2. Singer's conjecture is true for the case $n=5$ and the internal degree $15.2^{s}-5$ with $s$ an arbitrary non-negative integer.

Recall that the iterated Kameko homomorphism

$$
\left(\widetilde{S q}_{*}^{0}\right)^{s-1}:\left(Q P_{5}\right)_{15.2^{s}-5} \longrightarrow\left(Q P_{5}\right)_{25}
$$

is an isomorphism of $G L_{5}$-modules for every $s \geqslant 1$. Hence, we need only to prove Theorem 5.1 for $s=0,1$.

We need a notation for the proof of the theorem. For a fixed weight vector $\omega$, for any monomials $z_{1}, z_{2}, \ldots, z_{m}$ in $P_{n}(\omega)$ and for a subgroup $G \subset G L_{n}$, we denote $G\left(z_{1}, z_{2}, \ldots, z_{m}\right)$ the $G$-submodule of $Q P_{n}(\omega)$ generated by the set $\left\{\left[z_{i}\right]_{\omega}: 1 \leqslant i \leqslant\right.$ $m\}$.

\subsection{The case $s=0$.}

Denote by $a_{t}=a_{10, t}, 1 \leqslant t \leqslant 280$, the admissible monomials of degree 10 as given in Subsection 6.1. We have

$$
\left(Q P_{5}\right)_{10} \cong Q P_{5}(2,2,1) \oplus Q P_{5}(2,4) \oplus Q P_{5}(4,1,1) \oplus Q P_{5}(4,3) .
$$

We need some lemmas. 
Lemma 5.1.1. For $\omega=(2,2,1)$, we have $\operatorname{dim}\left(Q P_{5}^{0}(\omega)\right)^{\Sigma_{5}}=4$.

Proof. Observe that $\omega=(2,2,1)$ is the weight vector of the mimimal spike $x_{1}^{7} x_{2}^{3}$, hence $[f]_{\omega}=[f]$ for all $f \in P_{5}$. We have a direct summand decomposition of the $\Sigma_{5}$-modules

$$
Q P_{5}^{0}(\omega)=\Sigma_{5}\left(a_{1}\right) \oplus \Sigma_{5}\left(a_{21}\right) \oplus \Sigma_{5}\left(a_{51}\right) \oplus \Sigma_{5}\left(a_{116}\right)
$$

We prove the following:

$$
\begin{aligned}
\Sigma_{5}\left(a_{1}\right)^{\Sigma_{5}} & =\left\langle\left[p_{1}:=a_{1}+a_{2}+\ldots+a_{20}\right]\right\rangle, \\
\Sigma_{5}\left(a_{21}\right)^{\Sigma_{5}} & =\left\langle\left[p_{2}:=a_{21}+a_{22} \ldots+a_{50}\right]\right\rangle, \\
\Sigma_{5}\left(a_{51}\right)^{\Sigma_{5}} & =\left\langle\left[p_{3}:=a_{51}+a_{52}+\cdots+a_{90}\right]\right\rangle, \\
\Sigma_{5}\left(a_{126}\right)^{\Sigma_{5}} & =\left\langle\left[p_{4}:=a_{101}+a_{102}+\cdots+a_{145}\right]\right\rangle .
\end{aligned}
$$

For simplicity, we prove (5.2) in detail. The others can be proved by a similar computation. It is easy to see that $\Sigma_{5}\left(a_{21}\right)=\left\langle\left[a_{t}\right]: 21 \leqslant t \leqslant 50\right\rangle$. Let $[g] \in$ $\Sigma_{5}\left(a_{21}\right)^{\Sigma_{5}}$ with $g=\sum_{t=21}^{50} \gamma_{t} a_{t}$ and $\gamma_{t} \in \mathbb{F}_{2}$. Computing directly $\rho_{j}(g)+g$ in terms of $a_{t}, 21 \leqslant t \leqslant 50\left(\bmod \left(P_{5}^{-}(\omega)\right)\right.$. From the relations $\rho_{j}(g)+g \equiv 0$ with $1 \leqslant j \leqslant 4$, we get $\gamma_{t}=\gamma_{1}$ for $21 \leqslant t \leqslant 50$. The lemma follows.

By an argument analogous to the previous one, we easily obtain the following.

Lemma 5.1.2. Denote $M=\left\langle\left[a_{t}\right]: 231 \leqslant t \leqslant 240\right\rangle$. Then, $M$ is an $\Sigma_{5}$-submodule of $\left(Q P_{5}^{+}\right)_{10}$ and $M^{\Sigma_{5}}=\left\langle\left[p_{5}:=a_{232}+a_{233}+a_{234}+a_{235}\right]\right\rangle$.

Lemma 5.1.3. For $\omega=(4,1,1)$, we have $\left(Q P_{5}(\omega)\right)^{G L_{5}}=\left\langle\left[p_{6}\right] \omega\right\rangle$, where

$$
p_{6}=a_{241}+a_{242}+a_{243}+a_{244}+a_{257}+a_{258}+a_{259}+a_{260} .
$$

Proof. We have $Q P_{5}(\omega)=\Sigma_{5}\left(a_{161}\right) \oplus \Sigma_{5}\left(a_{181}\right) \oplus \Sigma_{5}\left(a_{241}\right)$. A direct computation shows

$$
\begin{aligned}
& \Sigma_{5}\left(a_{241}\right)=\left\langle\left[a_{t}\right]_{\omega}: 241 \leqslant t \leqslant 260\right\rangle, \Sigma_{5}\left(a_{211}\right)^{\Sigma_{5}}=\left\langle\left[p_{6}\right]_{\omega}\right\rangle, \\
& \Sigma_{5}\left(a_{161}\right)=\left\langle\left[a_{t}\right]_{\omega}: 161 \leqslant t \leqslant 180\right\rangle, \Sigma_{5}\left(a_{211}\right)^{\Sigma_{5}}=\left\langle\left[p_{7}\right]_{\omega}\right\rangle, \\
& \Sigma_{5}\left(a_{181}\right)=\left\langle\left[a_{t}\right]_{\omega}: 181 \leqslant t \leqslant 210\right\rangle, \Sigma_{5}\left(a_{261}\right)^{\Sigma_{5}}=\left\langle\left[p_{8}\right]_{\omega}\right\rangle,
\end{aligned}
$$

where $p_{7}=\sum_{t=161}^{180} a_{t}, p_{8}=\sum_{t=181}^{210} a_{t}$. Suppose $[f]_{\omega} \in\left(Q P_{5}(\omega)\right)^{G L_{5}}$ with $f \in$ $P_{5}(\omega)$. Since $[f]_{\omega} \in\left(Q P_{5}(\omega)\right)^{\Sigma_{5}}$, we obtain, $f \equiv_{\omega} \gamma_{1} p_{6}+\gamma_{2} p_{7}+\gamma_{3} p_{8}$ with $\gamma_{1}, \gamma_{2}, \gamma_{3} \in$ $\mathbb{F}_{2}$. By a direct computation, we get

$$
\rho_{5}(f)+f \equiv{ }_{\omega} \gamma_{2} a_{161}+\gamma_{3} a_{181}+\text { other terms } \equiv_{\omega} 0 .
$$

From this we get $\gamma_{2}=\gamma_{3}=0$. The lemma is proved.

Lemma 5.1.4. $\left(Q P_{5}(4,3)\right)^{G L_{5}}=0$.

Proof. It is easy to see that $Q P_{5}(\omega)=\Sigma_{5}\left(a_{211}\right) \oplus \Sigma_{5}\left(a_{261}\right)$ with $\omega=(4,3)$. By a simple computation, we have

$$
\begin{aligned}
& \Sigma_{5}\left(a_{211}\right)=\left\langle\left[a_{t}\right]_{\omega}: 211 \leqslant t \leqslant 230\right\rangle, \Sigma_{5}\left(a_{211}\right)^{\Sigma_{5}}=\left\langle\left[p_{9}\right]_{\omega}\right\rangle, \\
& \Sigma_{5}\left(a_{261}\right)=\left\langle\left[a_{t}\right]_{\omega}: 261 \leqslant t \leqslant 280\right\rangle, \Sigma_{5}\left(a_{261}\right)^{\Sigma_{5}}=\left\langle\left[p_{10}\right]_{\omega}\right\rangle,
\end{aligned}
$$


where $p_{9}=\sum_{t=211}^{230} a_{t}, p_{10}=\sum_{t=261}^{280} a_{t}$. Let $[f]_{\omega} \in\left(Q P_{5}(4,3)\right)^{G L_{5}}$ with $f \in$ $P_{5}(4,3)$, then $[f]_{\omega} \in\left(Q P_{5}(4,3)\right)^{\Sigma_{5}}$. Hence, $f \equiv_{\omega} \gamma_{1} p_{9}+\gamma_{2} p_{10}$ with $\gamma_{1}, \gamma_{2} \in \mathbb{F}_{2}$. By a direct computation, we get

$$
\rho_{5}(f)+f \equiv_{\omega} \gamma_{1} a_{211}+\gamma_{2} a_{267}+\text { other terms } \equiv_{\omega} 0 .
$$

This equality implies $\gamma_{1}=\gamma_{2}=0$. The lemma follows.

Proof of Theoren 5.1 for $s=0$. Let $[f] \in\left(Q P_{5}\right)_{10}^{G L}$. From Lemmas 5.1.4 and 5.1.3 we have $f \equiv f^{\prime}+\gamma_{6} p_{6}$ with $f^{\prime} \in P_{5}^{-}(4,1,1)$. A simple computation shows that $\left[p_{6}\right] \in$ $\left(Q P_{5}\right)_{10}^{\Sigma_{5}}$, hence $\left[f^{\prime}\right]$ is an $\Sigma_{5}$-invariant. Since $\left[P_{5}^{-}(4,1,1)\right]=\left(Q P_{5}^{0}(2,2,1) \oplus M\right.$, using Lemmas 5.1.1 and 5.1.2 we obtain $f^{\prime} \equiv \sum_{j=1}^{5} \gamma_{j} p_{j}$ with $\gamma_{j} \in \mathbb{F}_{2}$. By computing $\rho_{5}(f)+f$ in terms of the admissible monomials, we get

$$
\begin{aligned}
\rho_{5}(f)+f \equiv \gamma_{1} a_{15}+\gamma_{2} a_{24}+\gamma_{3} a_{56}+\gamma_{4} a_{92} \\
+\left(\gamma_{4}+\gamma_{5}\right) a_{119}+\gamma_{6} a_{239}+\text { other terms } \equiv 0 .
\end{aligned}
$$

From this it implies $\gamma_{j}=0,1 \leqslant j \leqslant 6$. Theorem 5.1 is true for $s=0$.

\subsection{The case $s=1$.}

Recall that Kameko's homomorphism $\left(\widetilde{S q}_{*}^{0}\right)_{(5,10)}:\left(Q P_{5}\right)_{25} \longrightarrow\left(Q P_{5}\right)_{10}$ is an epimorphism of $G L_{5}$-modules, hence from Theorem [5.1 for $s=0$, we need only to compute $\operatorname{Ker}\left(\widetilde{S q}_{*}^{0}\right)_{(5,10)}^{G L_{5}}$.

From the results in Section 4 we see that

$$
\operatorname{Ker}\left(\widetilde{S q}_{*}^{0}\right)_{(5,10)}=Q P_{5}(3,3,2,1)=Q P_{5}^{0}(3,3,2,1) \oplus Q P_{5}^{+}(3,3,2,1)
$$

where $\operatorname{dim} Q P_{5}^{0}(3,3,2,1)=520$ and $\operatorname{dim} Q P_{5}^{+}(3,3,2,1)=440$. Denote by $a_{t}=$ $a_{25, t}, 1 \leqslant t \leqslant 520$, the admissible monomials in $\left(P_{5}^{0}\right)_{25}$ as given in Subsection 6.3 By a direct computation, we can see that

$$
\begin{aligned}
\Sigma_{5}\left(a_{1}\right) & =\left\langle\left[a_{t}\right]: 1 \leqslant t \leqslant 60\right\rangle, \\
\Sigma_{5}\left(a_{61}\right) & =\left\langle\left[a_{t}\right]: 61 \leqslant t \leqslant 80\right\rangle, \\
\Sigma_{5}\left(a_{81}\right) & =\left\langle\left[a_{t}\right]: 81 \leqslant t \leqslant 140\right\rangle, \\
\Sigma_{5}\left(a_{141}\right) & =\left\langle\left[a_{t}\right]: 141 \leqslant t \leqslant 240\right\rangle,
\end{aligned}
$$

and $M_{1}=\left\langle\left[a_{t}\right]: 241 \leqslant t \leqslant 520\right\rangle$ are $\Sigma_{5}$-submodules of $Q P_{5}^{0}(3,3,2,1)$. Then, we have a direct summand decomposition of $\Sigma_{5}$-modules:

$$
Q P_{5}^{0}(3,3,2,1)=\Sigma_{5}\left(a_{1}\right) \oplus \Sigma_{5}\left(a_{61}\right) \oplus \Sigma_{5}\left(a_{81}\right) \oplus \Sigma_{5}\left(a_{141}\right) \oplus M_{1} .
$$

Lemma 5.2.1. We have

$$
\begin{aligned}
& \operatorname{dim} \Sigma_{5}\left(a_{1}\right)^{\Sigma_{5}}=\operatorname{dim} \Sigma_{5}\left(a_{81}\right)^{\Sigma_{5}}=\operatorname{dim} \Sigma_{5}\left(a_{141}\right)^{\Sigma_{5}}=1, \\
& \Sigma_{5}\left(a_{61}^{\Sigma_{5}}\right)=0, \operatorname{dim} M_{1}^{\Sigma_{5}}=3 .
\end{aligned}
$$

Outline of the proof. The set $A:=\left\{\left[a_{t}\right]: 1 \leqslant t \leqslant 60\right\}$ is a basis of $\left\langle\left[\Sigma_{5}\left(a_{1}\right)\right]\right\rangle$. The action of $\Sigma_{5}$ on $Q P_{5}$ induces the one of it on $A$. Furthermore, this action is transitive. Hence, if $f=\sum_{t=1}^{60} \gamma_{t} a_{t}$ with $\gamma_{t} \in \mathbb{F}_{2}$ and $[f] \in\left\langle\left[\Sigma_{5}\left(a_{1}\right)\right]\right\rangle^{\Sigma_{5}}$, then the relations $\rho_{j}(f) \equiv f, j=1,2,3,4$, imply $\gamma_{t}=\gamma_{1}, \forall t, 1 \leqslant t \leqslant 60$. Hence, $\Sigma_{5}\left(a_{1}\right)^{\Sigma_{5}}=\left\langle\left[q_{1}\right]\right\rangle$ with $q_{1}=\sum_{t=1}^{60} a_{t}$. Similarly, we have $\Sigma_{5}\left(a_{81}\right)^{\Sigma_{5}}=\left\langle\left[q_{2}\right]\right\rangle$ with $q_{2}=\sum_{t=81}^{140} a_{t}$. 
Suppose $g=\sum_{t=141}^{240} a_{t}$ and $[g] \in \Sigma_{5}\left(a_{141}\right)^{\Sigma_{5}}$. We compute $\rho_{j}(g)+g$ in terms of $a_{t}, 141 \leqslant t \leqslant 240$. By computing directly from the relations $\rho_{j}(g)+g \equiv 0$, $j=1,2,3,4$, we obtain $\gamma_{t}=0$ for $221 \leqslant t \leqslant 240$ and $\gamma_{t}=\gamma_{141}$ for $141 \leqslant t \leqslant 220$. Hence $\Sigma_{5}\left(a_{141}\right)^{\Sigma_{5}}=\left\langle\left[q_{3}\right]\right\rangle$ with $q_{3}=\sum_{t=141}^{220} a_{t}$. By a similar computation, we get $\Sigma_{5}\left(a_{61}\right)^{\Sigma_{5}}=0$.

Suppose $g=\sum_{t=241}^{520} \gamma_{t} a_{t}$ and $[g] \in M_{1}^{\Sigma_{5}}$. By computing $\rho_{j}(g)+g$ in terms of $a_{t}, 241 \leqslant t \leqslant 520$ and using the relations $\rho_{j}(g)+g \equiv 0, j=1,2,3,4$, one gets

$$
\left\{\begin{array}{l}
\gamma_{t}=0, \text { for } 486 \leqslant t \leqslant 520, \gamma_{t}=\gamma_{241} \text { for } 241 \leqslant t \leqslant 330 \\
\gamma_{t}=\gamma_{331} \text { for } 331 \leqslant t \leqslant 350, \gamma_{t}=\gamma_{351} \text { for } 351 \leqslant t \leqslant 400 \\
\gamma_{t}=\gamma_{401} \text { for } 401 \leqslant t \leqslant 425, \gamma_{t}=\gamma_{426} \text { for } 426 \leqslant t \leqslant 465 \\
\gamma_{t}=\gamma_{466} \text { for } 466 \leqslant t \leqslant 480, \gamma_{t}=\gamma_{351} \text { for } 481 \leqslant t \leqslant 485 \\
\gamma_{241}+\gamma_{351}+\gamma_{401}=\gamma_{241}+\gamma_{331}+\gamma_{426}=0 \\
\gamma_{241}+\gamma_{331}+\gamma_{351}+\gamma_{466}=\gamma_{331}+\gamma_{351}+\gamma_{481}=0
\end{array}\right.
$$

Set $q_{4}=\sum_{t=141}^{330} a_{t}+\sum_{t=401}^{480} a_{t}, q_{5}=\sum_{t=331}^{350} a_{t}+\sum_{t=426}^{485} a_{t}, q_{6}=\sum_{t=351}^{425} a_{t}+$ $\sum_{t=466}^{485} a_{t}$. From (5.5), we obtain $M_{1}^{\Sigma_{5}}=\left\langle\left[q_{4}\right],\left[q_{5}\right],\left[q_{6}\right]\right\rangle$. The lemma follows.

Denote by $b_{u}=b_{25, u}, 1 \leqslant u \leqslant 440$, the admissible monomials in $\left(P_{5}^{+}\right)_{25}$ as given in Subsections [6.4. It is easy to see that $\Sigma_{5}\left(b_{1}\right)=\left\langle\left[b_{u}\right]: 1 \leqslant u \leqslant 60\right\rangle$, $M_{2}=\left\langle\left[b_{u}\right]: 60 \leqslant u \leqslant 440\right\rangle$ are $\Sigma_{5}$-submodules of $Q P_{5}^{+}(3,3,2,1)$ and we have a direct summand decomposition of $\Sigma_{5}$-modules: $Q P_{5}^{+}(3,3,2,1)=\Sigma_{5}\left(b_{1}\right) \oplus M_{2}$.

Lemma 5.2.2. We have $\operatorname{dim} \Sigma_{5}\left(b_{1}\right)^{\Sigma_{5}}=1$ and $\operatorname{dim} M_{2}^{\Sigma_{5}}=3$.

Outline of the proof. Suppose $f=\sum_{u=1}^{60} b_{u}$ and $[f] \in \Sigma_{5}\left(b_{1}\right)^{\Sigma_{5}}$. A direct computation from the relations $\rho_{j}(f)+f \equiv 0, j=1,2,3,4$, shows that $\gamma_{u}=0$ for $46 \leqslant u \leqslant 60$ and $\gamma_{u}=\gamma_{1}$ for $1 \leqslant u \leqslant 45$. Hence $\Sigma_{5}\left(b_{1}\right)^{\Sigma_{5}}=\left\langle\left[q_{7}\right]\right\rangle$ with $q_{7}=\sum_{u=1}^{45} b_{u}$.

Suppose $g=\sum_{u=61}^{440} \gamma_{u} b_{u}$ and $[g] \in M_{2}^{\Sigma_{5}}$. We compute $\rho_{j}(g)+g$ in terms of $b_{u}, 61 \leqslant u \leqslant 440$. By using the relations $\rho_{j}(g)+g \equiv 0, j=1,2,3,4$, we have

$$
\left\{\begin{array}{l}
\gamma_{u}=0, \text { for } 267 \leqslant u \leqslant 440, \gamma_{u}=\gamma_{61} \text { for } 61 \leqslant u \leqslant 125 \\
\gamma_{u}=\gamma_{126} \text { for } 126 \leqslant u \leqslant 175, \gamma_{u}=\gamma_{176} \text { for } 176 \leqslant u \leqslant 226 \\
\gamma_{u}=\gamma_{227} \text { for } 227 \leqslant u \leqslant 258, \gamma_{u}=\gamma_{259} \text { for } 259 \leqslant u \leqslant 262, \\
\gamma_{263}=\gamma_{264}=\gamma_{265}, \gamma_{61}+\gamma_{126}+\gamma_{227}=\gamma_{126}+\gamma_{176}+\gamma_{259}=0 \\
\gamma_{61}+\gamma_{126}+\gamma_{176}+\gamma_{263}=\gamma_{61}+\gamma_{176}+\gamma_{266}=0 .
\end{array}\right.
$$

Set $q_{8}=\sum_{u=61}^{125} b_{u}+\sum_{u=227}^{258} b_{u}+\sum_{u=263}^{266} b_{u}, q_{9}=\sum_{u=126}^{175} b_{u}+\sum_{u=226}^{265} b_{u}, q_{10}=$ $\sum_{u=176}^{226} b_{u}+\sum_{u=259}^{266} b_{u}$. From (5.6), we obtain $M_{2}^{\Sigma_{5}}=\left\langle\left[q_{8}\right],\left[q_{9}\right],\left[q_{10}\right]\right\rangle$. The lemma is proved.

Combining Lemmas 5.2.1 and 5.2 .2 one gets the following.

Corollary 5.2.3. $\operatorname{dim} \operatorname{Ker}\left(\widetilde{S q}_{*}^{0}\right)_{(5,10)}^{\Sigma_{5}}=10$.

Proof of Theorem 5.1 for $s=1$. Suppose $[h] \in \operatorname{Ker}\left(\widetilde{S q}_{*}^{0}\right)_{(5,10)}^{G L_{5}}$ with $h$ a polynomial in $\left(P_{5}\right)_{25}$. Since $[h] \in \operatorname{Ker}\left(\widetilde{S q}_{*}^{0}\right)_{(5,10)}^{\Sigma_{5}}$, from Lemmas [5.2.1] and 5.2.2, we have 
$h \equiv \sum_{j=1}^{10} \delta_{j} q_{j}$ with $\delta_{j} \in \mathbb{F}_{2}$. By computing $\rho_{5}(h)+h$ in terms of the admissible monomials, we get

$$
\begin{aligned}
\rho_{5}(h)+h \equiv & \delta_{1} a_{31}+\delta_{2} a_{81}+\delta_{3} a_{144}+\delta_{4} a_{234}+\left(\delta_{5}+\delta_{10}\right) a_{331}+\delta_{6} a_{263} \\
& +\delta_{7} b_{19}+\delta_{8} b_{78}+\delta_{9} b_{138}+\delta_{10} b_{187}+\text { other terms } \equiv 0 .
\end{aligned}
$$

The last equality implies that $\gamma_{j}=0,1 \leqslant j \leqslant 6$. The proof is completed.

\section{Appendix}

In this section, we list the admissible monomials of degrees 10, 11, 25 in $P_{5}$.

\subsection{The admissible monomials of degree $\mathbf{1 0}$ in $P_{5}$.}

$B_{5}^{0}(10)$ is the set of 230 monomials: $a_{10, t}, 1 \leqslant t \leqslant 230$.

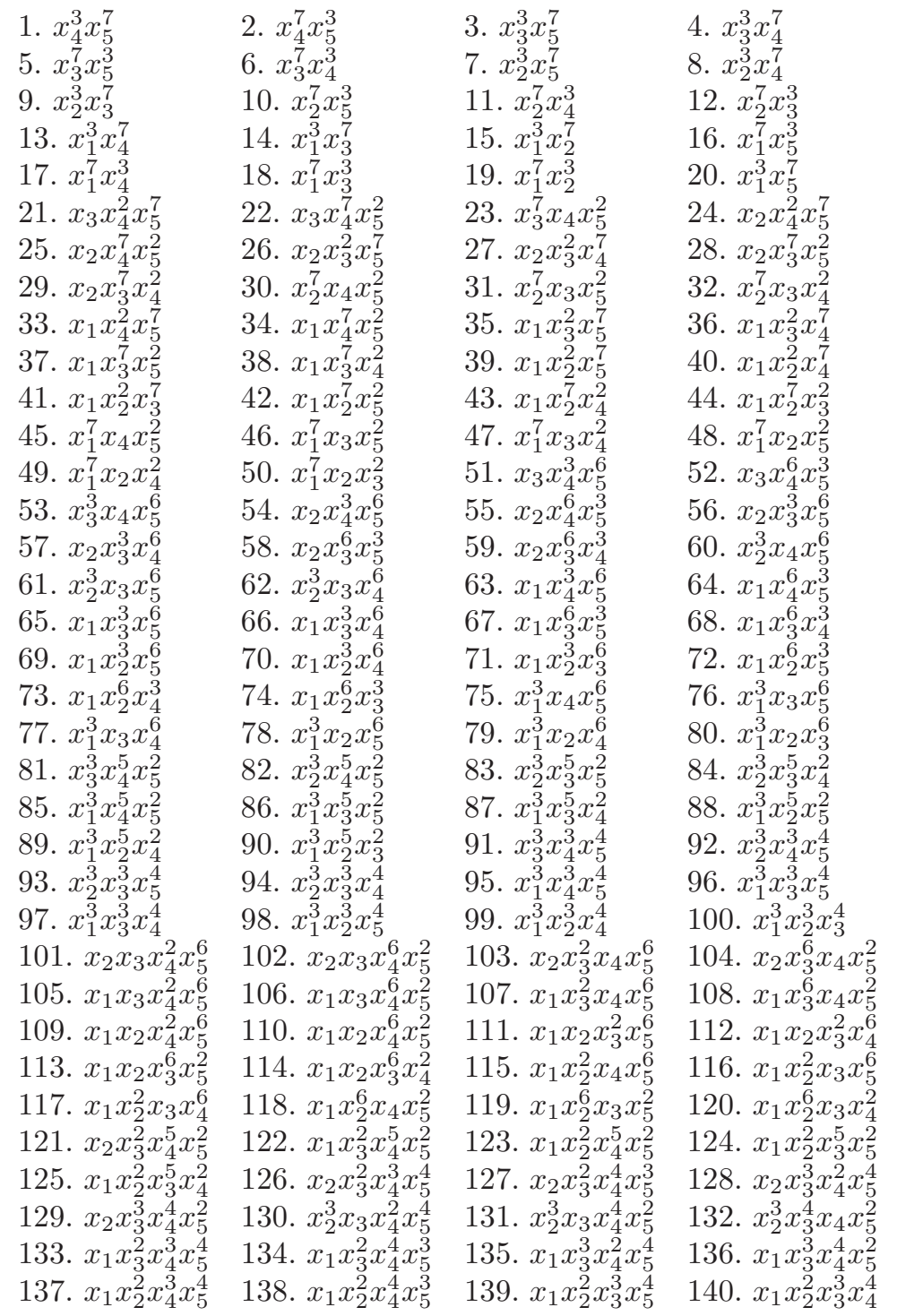




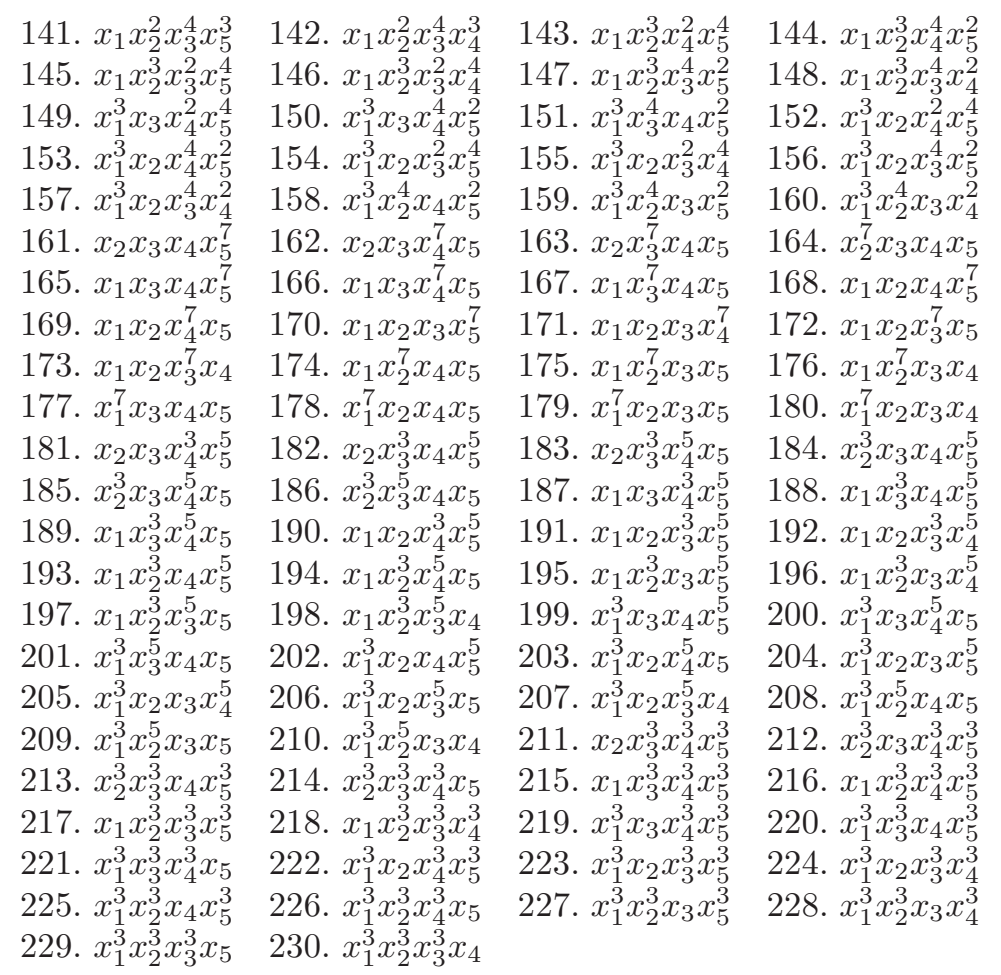

We have $B_{5}^{+}(10)=B_{5}^{+}(2,2,1) \cup B_{5}(2,4) \cup B_{5}^{+}(4,1,1) \cup B_{5}^{+}(4,3)$

$B_{5}^{+}(2,2,1)$ is the set of 5 monomials: $a_{10, t}, 231 \leqslant t \leqslant 235$.
231. $x_{1} x_{2} x_{3}^{2} x_{4}^{2} x_{5}^{4}$
232. $x_{1} x_{2} x_{3}^{2} x_{4}^{4} x_{5}^{2}$
233. $x_{1} x_{2}^{2} x_{3} x_{4}^{2} x_{5}^{4}$
234. $x_{1} x_{2}^{2} x_{3} x_{4}^{4} x_{5}^{2}$
235. $x_{1} x_{2}^{2} x_{3}^{4} x_{4} x_{5}^{2}$

$B_{5}^{+}(2,4)$ is the set of 5 monomials: $a_{10, t}, 236 \leqslant t \leqslant 240$.

$\begin{array}{llll}236 . x_{1} x_{2}^{2} x_{3}^{2} x_{4}^{2} x_{5}^{3} & \text { 237. } x_{1} x_{2}^{2} x_{3}^{2} x_{4}^{3} x_{5}^{2} & \text { 238. } x_{1} x_{2}^{2} x_{3}^{3} x_{4}^{2} x_{5}^{2} & \text { 239. } x_{1} x_{2}^{3} x_{3}^{2} x_{4}^{2} x_{5}^{2}\end{array}$ 240. $x_{1}^{3} x_{2} x_{3}^{2} x_{4}^{2} x_{5}^{2}$

$B_{5}^{+}(4,1,1)$ is the set of 20 monomials: $a_{10, t}, 241 \leqslant t \leqslant 260$.
241. $x_{1} x_{2} x_{3} x_{4} x_{5}^{6}$
242. $x_{1} x_{2} x_{3} x_{4}^{6} x_{5}$
243. $x_{1} x_{2} x_{3}^{6} x_{4} x_{5}$
244. $x_{1} x_{2}^{6} x_{3} x_{4} x_{5}$
245. $x_{1} x_{2} x_{3} x_{4}^{2} x_{5}^{5}$
246. $x_{1} x_{2} x_{3}^{2} x_{4} x_{5}^{5}$
247. $x_{1} x_{2} x_{3}^{2} x_{4}^{5} x_{5}$
248. $x_{1} x_{2}^{2} x_{3} x_{4} x_{5}^{5}$
249. $x_{1} x_{2}^{2} x_{3} x_{4}^{5} x_{5}$
250. $x_{1} x_{2}^{2} x_{3}^{5} x_{4} x_{5}$
251. $x_{1} x_{2} x_{3} x_{4}^{3} x_{5}^{4}$
252. $x_{1} x_{2} x_{3}^{3} x_{4} x_{5}^{4}$
253. $x_{1} x_{2} x_{3}^{3} x_{4}^{4} x_{5}$
254. $x_{1} x_{2}^{3} x_{3} x_{4} x_{5}^{4}$
255. $x_{1} x_{2}^{3} x_{3} x_{4}^{4} x_{5}$
256. $x_{1} x_{2}^{3} x_{3}^{4} x_{4} x_{5}$
257. $x_{1}^{3} x_{2} x_{3} x_{4} x_{5}^{4}$
258. $x_{1}^{3} x_{2} x_{3} x_{4}^{4} x_{5} \quad$ 259. $x_{1}^{3} x_{2} x_{3}^{4} x_{4} x_{5}$
260. $x_{1}^{3} x_{2}^{4} x_{3} x_{4} x_{5}$

$B_{5}^{+}(4,3)$ is the set of 20 monomials: $a_{10, t}, 261 \leqslant t \leqslant 280$.
261. $x_{1} x_{2} x_{3}^{2} x_{4}^{3} x_{5}^{3}$
265. $x_{1} x_{2}^{2} x_{3}^{3} x_{4} x_{5}^{3}$
269. $x_{1} x_{2}^{3} x_{3}^{2} x_{4} x_{5}^{3}$
262. $x_{1} x_{2} x_{3}^{3} x_{4}^{2} x_{5}^{3}$
266. $x_{1} x_{2}^{2} x_{3}^{3} x_{4}^{3} x_{5}$
263. $x_{1} x_{2} x_{3}^{3} x_{4}^{3} x_{5}^{2}$
270. $x_{1} x_{2}^{3} x_{3}^{2} x_{4}^{3} x_{5}$
267. $x_{1} x_{2}^{3} x_{3} x_{4}^{2} x_{5}^{3}$
273. $x_{1}^{3} x_{2} x_{3} x_{4}^{2} x_{5}^{3}$
274. $x_{1}^{3} x_{2} x_{3} x_{4}^{3} x_{5}^{2}$
271. $x_{1} x_{2}^{3} x_{3}^{3} x_{4} x_{5}^{2}$
275. $x_{1}^{3} x_{2} x_{3}^{2} x_{4} x_{5}^{3}$
277. $x_{1}^{3} x_{2} x_{3}^{3} x_{4} x_{5}^{2}$
278. $x_{1}^{3} x_{2} x_{3}^{3} x_{4}^{2} x_{5}$
279. $x_{1}^{3} x_{2}^{3} x_{3} x_{4} x_{5}^{2}$
264. $x_{1} x_{2}^{2} x_{3} x_{4}^{3} x_{5}^{3}$
268. $x_{1} x_{2}^{3} x_{3} x_{4}^{3} x_{5}^{2}$
272. $x_{1} x_{2}^{3} x_{3}^{3} x_{4}^{2} x_{5}$
276. $x_{1}^{3} x_{2} x_{3}^{2} x_{4}^{3} x_{5}$
280. $x_{1}^{3} x_{2}^{3} x_{3} x_{4}^{2} x_{5}$ 
By a simple computation using the results in [26], we have

$$
\begin{aligned}
B_{5}(2,2,1) & =\Phi\left(B_{4}(2,2,1)\right), \\
B_{5}(4,1,1) & =\Phi\left(B_{4}(4,1,1)\right) \cup\left\{x_{1}^{3} x_{2}^{4} x_{3} x_{4} x_{5}\right\}, \\
B_{5}(4,3) & =\Phi\left(B_{4}(4,3)\right) .
\end{aligned}
$$

Combining this and the fact that $B_{4}(2,4)=\emptyset$ we see that Conjecture 3.4 is true for $n=5$ and the degree 10 .

\subsection{The admissible monomials of degree 11 in $P_{5}$.}

$B_{5}^{0}(11)$ is the set of 240 monomials:

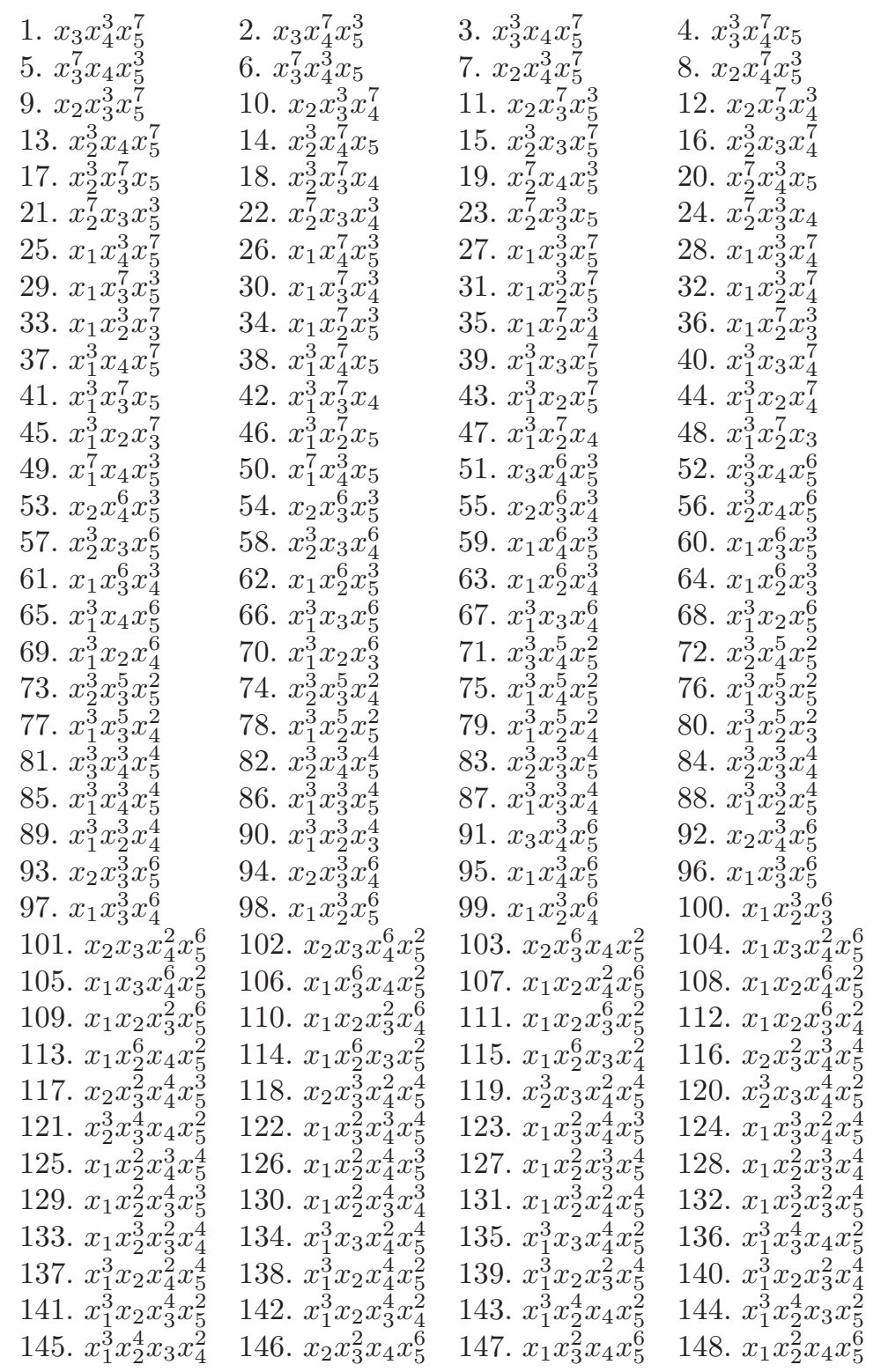




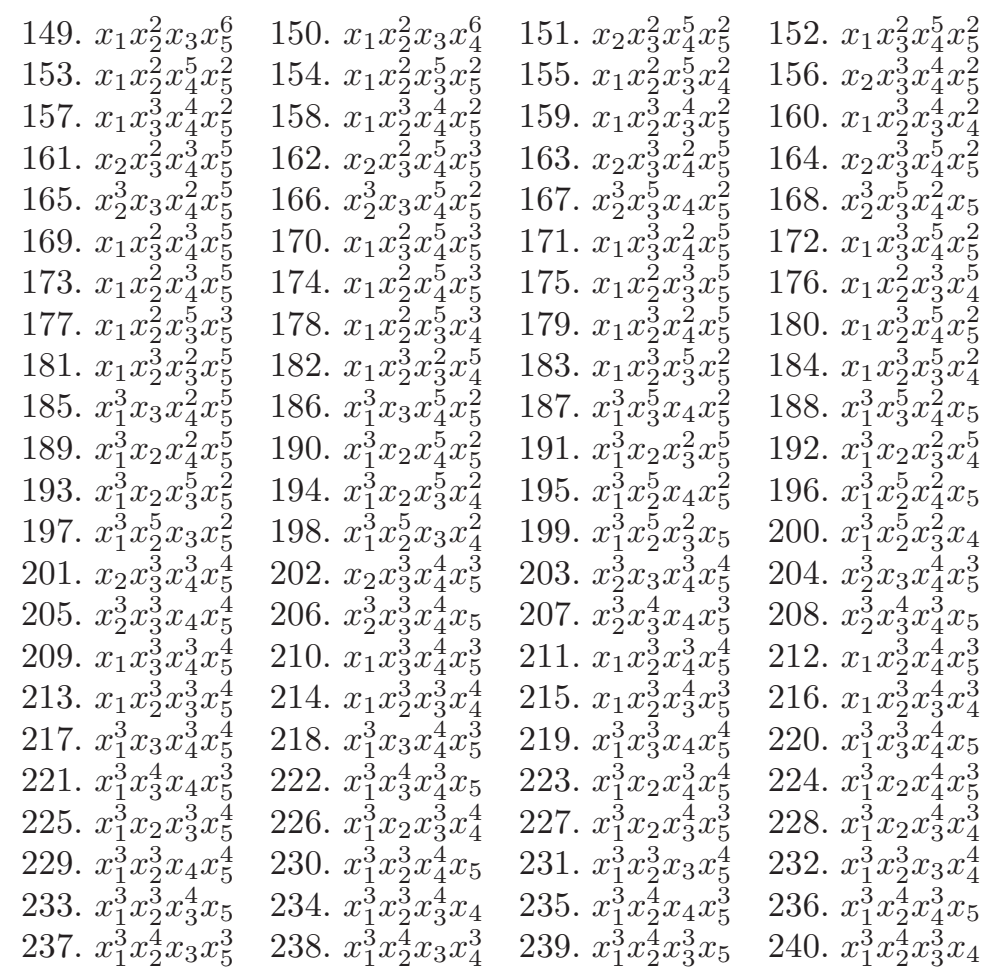

We have $B_{5}^{+}(11)=B_{5}^{+}(3,2,1) \cup B_{5}(3,4) \cup \psi\left(B_{5}(3)\right)$.

$B_{5}^{+}(3,2,1)$ is the set of 40 monomials:
1. $x_{1} x_{2} x_{3} x_{4}^{2} x_{5}^{6}$
2. $x_{1} x_{2} x_{3} x_{4}^{6} x_{5}^{2}$
3. $x_{1} x_{2} x_{3}^{2} x_{4} x_{5}^{6}$
4. $x_{1} x_{2} x_{3}^{2} x_{4}^{2} x_{5}^{5}$
5. $x_{1} x_{2} x_{3}^{2} x_{4}^{3} x_{5}^{4}$
6. $x_{1} x_{2} x_{3}^{2} x_{4}^{4} x_{5}^{3}$
7. $x_{1} x_{2} x_{3}^{2} x_{4}^{5} x_{5}^{2}$
9. $x_{1} x_{2} x_{3}^{3} x_{4}^{2} x_{5}^{4}$
10. $x_{1} x_{2} x_{3}^{3} x_{4}^{4} x_{5}^{2}$
11. $x_{1} x_{2} x_{3}^{6} x_{4} x_{5}^{2}$
8. $x_{1} x_{2} x_{3}^{2} x_{4}^{6} x_{5}$
13. $x_{1} x_{2}^{2} x_{3} x_{4} x_{5}^{6}$
14. $x_{1} x_{2}^{2} x_{3} x_{4}^{2} x_{5}^{5}$
15. $x_{1} x_{2}^{2} x_{3} x_{4}^{3} x_{5}^{4}$
12. $x_{1} x_{2} x_{3}^{6} x_{4}^{2} x_{5}$
17. $x_{1} x_{2}^{2} x_{3} x_{4}^{5} x_{5}^{2}$
18. $x_{1} x_{2}^{2} x_{3} x_{4}^{6} x_{5}$
19. $x_{1} x_{2}^{2} x_{3}^{3} x_{4} x_{5}^{4}$
16. $x_{1} x_{2}^{2} x_{3} x_{4}^{4} x_{5}^{3}$
21. $x_{1} x_{2}^{2} x_{3}^{4} x_{4} x_{5}^{3}$
22. $x_{1} x_{2}^{2} x_{3}^{4} x_{4}^{3} x_{5}$
23. $x_{1} x_{2}^{2} x_{3}^{5} x_{4} x_{5}^{2}$
20. $x_{1} x_{2}^{2} x_{3}^{3} x_{4}^{4} x_{5}$
25. $x_{1} x_{2}^{3} x_{3} x_{4}^{2} x_{5}^{4}$
26. $x_{1} x_{2}^{3} x_{3} x_{4}^{4} x_{5}^{2}$
27. $x_{1} x_{2}^{3} x_{3}^{2} x_{4} x_{5}^{4}$
24. $x_{1} x_{2}^{2} x_{3}^{5} x_{4}^{2} x_{5}$
29. $x_{1} x_{2}^{3} x_{3}^{4} x_{4} x_{5}^{2}$
30. $x_{1} x_{2}^{3} x_{3}^{4} x_{4}^{2} x_{5}$
31. $x_{1} x_{2}^{6} x_{3} x_{4} x_{5}^{2}$
28. $x_{1} x_{2}^{3} x_{3}^{2} x_{4}^{4} x_{5}$
33. $x_{1}^{3} x_{2} x_{3} x_{4}^{2} x_{5}^{4}$
34. $x_{1}^{3} x_{2} x_{3} x_{4}^{4} x_{5}^{2}$
35. $x_{1}^{3} x_{2} x_{3}^{2} x_{4} x_{5}^{4}$
32. $x_{1} x_{2}^{6} x_{3} x_{4}^{2} x_{5}$
37. $x_{1}^{3} x_{2} x_{3}^{4} x_{4} x_{5}^{2}$
38. $x_{1}^{3} x_{2} x_{3}^{4} x_{4}^{2} x_{5}$
39. $x_{1}^{3} x_{2}^{4} x_{3} x_{4} x_{5}^{2}$
36. $x_{1}^{3} x_{2} x_{3}^{2} x_{4}^{4} x_{5}$
40. $x_{1}^{3} x_{2}^{4} x_{3} x_{4}^{2} x_{5}$

$B_{5}^{+}(3,4)$ is the set of 10 monomials:
41. $x_{1} x_{2}^{2} x_{3}^{2} x_{4}^{3} x_{5}^{3}$
45. $x_{1} x_{2}^{3} x_{3}^{2} x_{4}^{3} x_{5}^{2}$
49. $x_{1}^{3} x_{2} x_{3}^{3} x_{4}^{2} x_{5}^{2}$
42. $x_{1} x_{2}^{2} x_{3}^{3} x_{4}^{2} x_{5}^{3}$
43. $x_{1} x_{2}^{2} x_{3}^{3} x_{4}^{3} x_{5}^{2}$
44. $x_{1} x_{2}^{3} x_{3}^{2} x_{4}^{2} x_{5}^{3}$
47. $x_{1}^{3} x_{2} x_{3}^{2} x_{4}^{2} x_{5}^{3}$ 48. $x_{1}^{3} x_{2} x_{3}^{2} x_{4}^{3} x_{5}^{2}$

By a simple computation using the results in [26, we can easily show that $B_{5}(3,2,1)=\Phi\left(B_{4}(3,2,1)\right)$. Combining this and the fact that $B_{4}(3,4)=\emptyset$ we see that Conjecture 3.4 is true for $n=5$ and the degree 11 .

\subsection{The admissible monomials of degree 25 in $P_{5}^{0}$.}

We have $B_{5}(25)=B_{5}^{0}(25) \cup B_{5}^{+}(25)$.

$B_{5}^{0}(25)=B_{5}^{0}(3,3,2,1)$ is the set of 520 monomials $a_{25, t}, 1 \leqslant t \leqslant 520$. 


\begin{tabular}{|c|c|c|c|}
\hline $\begin{array}{l}\text { 1. } x_{3}^{3} x_{4}^{7} x_{5}^{15} \\
\text { 5. } x_{3}^{15} x_{4}^{3} x_{5}^{7} \\
\text { 9. } x_{2}^{3} x_{3}^{7} x_{5}^{15} \\
\text { 13. } x_{2}^{7} x_{4}^{3} x_{5}^{15} \\
\text { 17. } x_{2}^{7} x_{3}^{15} x_{5}^{3} \\
\text { 21. } x_{2}^{15} x_{3}^{3} x_{5}^{7} \\
\text { 25. } x_{1}^{3} x_{4}^{7} x_{5}^{15} \\
\text { 29. } x_{1}^{3} x_{3}^{15} x_{5}^{7} \\
\text { 33. } x_{1}^{3} x_{2}^{7} x_{3}^{15} \\
\text { 37. } x_{1}^{7} x_{4}^{3} x_{5}^{15} \\
\text { 41. } x_{1}^{7} x_{3}^{15} x_{5}^{3} \\
\text { 45. } x_{1}^{7} x_{2}^{3} x_{3}^{15} \\
\text { 49. } x_{1}^{15} x_{4}^{3} x_{5}^{7} \\
\text { 53. } x_{1}^{15} x_{3}^{7} x_{5}^{3} \\
\text { 57. } x_{1}^{15} x_{2}^{3} x_{3}^{7} \\
\text { 61. } x_{3}^{7} x_{4}^{7} x_{5}^{11} \\
\text { 65. } x_{2}^{7} x_{3}^{7} x_{5}^{11} \\
\text { 69. } x_{1}^{7} x_{4}^{7} x_{5}^{11} \\
\text { 73. } x_{1}^{7} x_{3}^{11} x_{5}^{7} \\
\text { 77. } x_{1}^{7} x_{2}^{7} x_{3}^{11} \\
\text { 81. } x_{2} x_{3}^{2} x_{4}^{7} x_{5}^{15} \\
\text { 85. } x_{2} x_{3}^{15} x_{4}^{2} x_{5}^{7} \\
\text { 89. } x_{2}^{7} x_{3}^{15} x_{4} x_{5}^{2} \\
\text { 93. } x_{1} x_{3}^{2} x_{4}^{7} x_{5}^{15} \\
\text { 97. } x_{1} x_{3}^{15} x_{4}^{2} x_{5}^{7} \\
\text { 101. } x_{1} x_{2}^{2} x_{3}^{7} x_{5}^{15} \\
\text { 105. } x_{1} x_{2}^{7} x_{4}^{2} x_{5}^{15} \\
\text { 109. } x_{1} x_{2}^{7} x_{3}^{15} x_{5}^{2} \\
\text { 113. } x_{1} x_{2}^{15} x_{3}^{2} x_{5}^{7} \\
\text { 117. } x_{1}^{7} x_{3} x_{4}^{2} x_{5}^{15} \\
\text { 121. } x_{1}^{7} x_{2} x_{4}^{15} x_{5}^{2} \\
\text { 125. } x_{1}^{7} x_{2} x_{3}^{15} x_{4}^{2} \\
\text { 129. } x_{1}^{15} x_{3} x_{4}^{2} x_{5}^{7} \\
\text { 133. } x_{1}^{15} x_{2} x_{4}^{7} x_{5}^{2} \\
\text { 137. } x_{1}^{15} x_{2} x_{3}^{7} x_{4}^{2} \\
\text { 141. } x_{2} x_{3}^{6} x_{4}^{3} x_{5}^{15} \\
\text { 145. } x_{2}^{3} x_{3} x_{4}^{15} x_{5}^{6} \\
\text { 149. } x_{2}^{3} x_{3}^{5} x_{4}^{15} x_{5}^{2} \\
\text { 153. } x_{2}^{15} x_{3} x_{4}^{6} x_{5}^{3} \\
\text { 157. } x_{1} x_{3}^{6} x_{4}^{3} x_{5}^{15} \\
\text { 161. } x_{1} x_{2}^{6} x_{4}^{15} x_{5}^{3} \\
\text { 165. } x_{1} x_{2}^{6} x_{3}^{15} x_{4}^{3} \\
\text { 169. } x_{1}^{3} x_{3} x_{4}^{6} x_{5}^{15} \\
\text { 173. } x_{1}^{3} x_{3}^{5} x_{4}^{2} x_{5}^{15} \\
\text { 177. } x_{1}^{3} x_{3}^{15} x_{4}^{5} x_{5}^{2} \\
\text { 181. } x_{1}^{3} x_{2} x_{3}^{6} x_{4}^{15} \\
\text { 185. } x_{1}^{3} x_{2}^{3} x_{4}^{15} x_{5}^{4} x_{3}^{3} x_{3}^{15} x_{4}^{4} \\
\text { 189. }\end{array}$ & $\begin{array}{l}\text { 2. } x_{3}^{3} x_{4}^{15} x_{5}^{7} \\
\text { 6. } x_{3}^{15} x_{4}^{7} x_{5}^{3} \\
\text { 10. } x_{2}^{3} x_{3}^{7} x_{4}^{15} \\
\text { 14. } x_{2}^{7} x_{4}^{15} x_{5}^{3} \\
\text { 18. } x_{2}^{7} x_{3}^{15} x_{4}^{3} \\
\text { 22. } x_{2}^{15} x_{3}^{3} x_{4}^{7} \\
\text { 26. } x_{1}^{3} x_{4}^{15} x_{5}^{7} \\
\text { 30. } x_{1}^{3} x_{3}^{15} x_{4}^{7} \\
\text { 34. } x_{1}^{3} x_{2}^{15} x_{5}^{7} \\
\text { 38. } x_{1}^{7} x_{4}^{15} x_{5}^{3} \\
\text { 42. } x_{1}^{7} x_{3}^{15} x_{4}^{3} \\
\text { 46. } x_{1}^{7} x_{2}^{15} x_{5}^{3} \\
\text { 50. } x_{1}^{15} x_{4}^{7} x_{5}^{3} \\
\text { 54. } x_{1}^{15} x_{3}^{7} x_{4}^{3} \\
\text { 58. } x_{1}^{15} x_{2}^{7} x_{5}^{3} \\
\text { 62. } x_{3}^{7} x_{4}^{11} x_{5}^{7} \\
\text { 66. } x_{2}^{7} x_{3}^{7} x_{4}^{11} \\
\text { 70. } x_{1}^{7} x_{4}^{11} x_{5}^{7} \\
\text { 74. } x_{1}^{7} x_{3}^{11} x_{4}^{7} \\
\text { 78. } x_{1}^{7} x_{2}^{11} x_{5}^{7} \\
\text { 82. } x_{2} x_{3}^{2} x_{4}^{15} x_{5}^{7} \\
\text { 86. } x_{2} x_{3}^{15} x_{4}^{7} x_{5}^{2} \\
\text { 90. } x_{2}^{15} x_{3} x_{4}^{2} x_{5}^{7} \\
\text { 94. } x_{1} x_{3}^{2} x_{4}^{15} x_{5}^{7} \\
\text { 98. } x_{1} x_{3}^{15} x_{4}^{7} x_{5}^{2} \\
\text { 102. } x_{1} x_{2}^{2} x_{3}^{7} x_{4}^{15} \\
\text { 106. } x_{1} x_{2}^{7} x_{4}^{15} x_{5}^{2} \\
\text { 110. } x_{1} x_{2}^{7} x_{3}^{15} x_{4}^{2} \\
\text { 114. } x_{1} x_{2}^{15} x_{3}^{2} x_{4}^{7} \\
\text { 118. } x_{1}^{7} x_{3} x_{4}^{15} x_{5}^{2} \\
\text { 122. } x_{1}^{7} x_{2} x_{3}^{2} x_{5}^{15} \\
\text { 126. } x_{1}^{7} x_{2}^{15} x_{4} x_{5}^{2} \\
\text { 130. } x_{1}^{15} x_{3} x_{4}^{7} x_{5}^{2} \\
\text { 134. } x_{1}^{15} x_{2} x_{3}^{2} x_{5}^{7} \\
\text { 138. } x_{1}^{15} x_{2}^{7} x_{4} x_{5}^{2} \\
\text { 142. } x_{2} x_{3}^{6} x_{4}^{15} x_{5}^{3} \\
\text { 146. } x_{2}^{3} x_{3}^{3} x_{4}^{4} x_{5}^{15} \\
\text { 150. } x_{2}^{3} x_{3}^{15} x_{4} x_{5}^{6} \\
\text { 154. } x_{2}^{15} x_{3}^{3} x_{4} x_{5}^{6} \\
\text { 158. } x_{1} x_{3}^{6} x_{4}^{15} x_{5}^{3} \\
\text { 162. } x_{1} x_{2}^{6} x_{3}^{3} x_{5}^{15} \\
\text { 166. } x_{1} x_{2}^{15} x_{4}^{6} x_{5}^{3} \\
\text { 170. } x_{1}^{3} x_{3} x_{4}^{15} x_{5}^{6} \\
\text { 174. } x_{1}^{3} x_{3}^{5} x_{4}^{15} x_{5}^{2} \\
\text { 178. } x_{1}^{3} x_{2} x_{4}^{6} x_{5}^{15} \\
\text { 182. } x_{1}^{3} x_{2} x_{3}^{15} x_{5}^{6} \\
\text { 186. } x_{1}^{3} x_{2}^{3} x_{3}^{4} x_{5}^{15} \\
\text { 190. } x_{1}^{3} x_{2}^{5} x_{4}^{2} x_{5}^{15}\end{array}$ & $\begin{array}{l}\text { 3. } x_{3}^{7} x_{4}^{3} x_{5}^{15} \\
\text { 7. } x_{2}^{3} x_{4}^{7} x_{5}^{15} \\
\text { 11. } x_{2}^{3} x_{3}^{15} x_{5}^{7} \\
\text { 15. } x_{2}^{7} x_{3}^{3} x_{5}^{15} \\
\text { 19. } x_{2}^{15} x_{4}^{3} x_{5}^{7} \\
\text { 23. } x_{2}^{15} x_{3}^{7} x_{5}^{3} \\
\text { 27. } x_{1}^{3} x_{3}^{7} x_{5}^{15} \\
\text { 31. } x_{1}^{3} x_{2}^{7} x_{5}^{15} \\
\text { 35. } x_{1}^{3} x_{2}^{15} x_{4}^{7} \\
\text { 39. } x_{1}^{7} x_{3}^{3} x_{5}^{15} \\
\text { 43. } x_{1}^{7} x_{2}^{3} x_{5}^{15} \\
\text { 47. } x_{1}^{7} x_{2}^{15} x_{4}^{3} \\
\text { 51. } x_{1}^{15} x_{3}^{3} x_{5}^{7} \\
\text { 55. } x_{1}^{15} x_{2}^{3} x_{5}^{7} \\
\text { 59. } x_{1}^{15} x_{2}^{7} x_{4}^{3} \\
\text { 63. } x_{2}^{7} x_{4}^{7} x_{5}^{11} \\
\text { 67. } x_{2}^{7} x_{3}^{11} x_{5}^{7} \\
\text { 71. } x_{1}^{7} x_{3}^{7} x_{5}^{11} \\
\text { 75. } x_{1}^{7} x_{2}^{7} x_{5}^{11} \\
\text { 79. } x_{1}^{7} x_{2}^{11} x_{4}^{7} \\
\text { 83. } x_{2} x_{3}^{7} x_{4}^{2} x_{5}^{15} \\
\text { 87. } x_{2}^{7} x_{3} x_{4}^{2} x_{5}^{15} \\
\text { 91. } x_{2}^{15} x_{3} x_{4}^{7} x_{5}^{2} \\
\text { 95. } x_{1} x_{3}^{7} x_{4}^{2} x_{5}^{15} \\
\text { 99. } x_{1} x_{2}^{2} x_{4}^{7} x_{5}^{15} \\
\text { 103. } x_{1} x_{2}^{2} x_{3}^{15} x_{5}^{7} \\
\text { 107. } x_{1} x_{2}^{7} x_{3}^{2} x_{5}^{15} \\
\text { 111. } x_{1} x_{2}^{15} x_{4}^{2} x_{5}^{7} \\
\text { 115. } x_{1} x_{2}^{15} x_{3}^{7} x_{5}^{2} \\
\text { 119. } x_{1}^{7} x_{3}^{15} x_{4} x_{5}^{2} \\
\text { 123. } x_{1}^{7} x_{2} x_{3}^{2} x_{4}^{15} \\
\text { 127. } x_{1}^{7} x_{2}^{15} x_{3} x_{5}^{2} \\
\text { 131. } x_{1}^{15} x_{3}^{7} x_{4} x_{5}^{2} \\
\text { 135. } x_{1}^{15} x_{2} x_{3}^{2} x_{4}^{7} \\
\text { 139. } x_{1}^{15} x_{2}^{7} x_{3} x_{5}^{2} \\
\text { 143. } x_{2} x_{3}^{15} x_{4}^{6} x_{5}^{3} \\
\text { 147. } x_{2}^{3} x_{3}^{3} x_{4}^{15} x_{5}^{4} \\
\text { 151. } x_{2}^{3} x_{3}^{15} x_{4}^{3} x_{5}^{4} \\
\text { 155. } x_{2}^{15} x_{3}^{3} x_{4}^{3} x_{5}^{4} \\
\text { 159. } x_{1} x_{3}^{15} x_{4}^{6} x_{5}^{3} \\
\text { 163. } x_{1} x_{2}^{6} x_{3}^{3} x_{4}^{15} \\
\text { 167. } x_{1} x_{2}^{11} x_{3}^{6} x_{5}^{3} \\
\text { 171. } x_{1}^{3} x_{3}^{3} x_{4}^{4} x_{5}^{15} \\
\text { 175. } x_{1}^{3} x_{3}^{15} x_{4} x_{5}^{6} \\
\text { 179. } x_{1}^{3} x_{2} x_{4}^{15} x_{5}^{6} \\
\text { 183. } x_{1}^{3} x_{2} x_{3}^{15} x_{4}^{6} \\
\text { 187. } x_{1}^{3} x_{2}^{3} x_{3}^{4} x_{4}^{15} \\
\text { 191. } x_{1}^{3} x_{2}^{5} x_{4}^{15} x_{5}^{2}\end{array}$ & 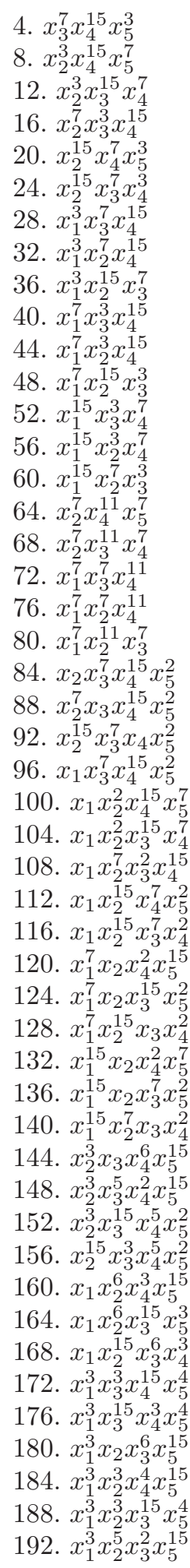 \\
\hline
\end{tabular}




\begin{tabular}{|c|c|c|c|}
\hline & 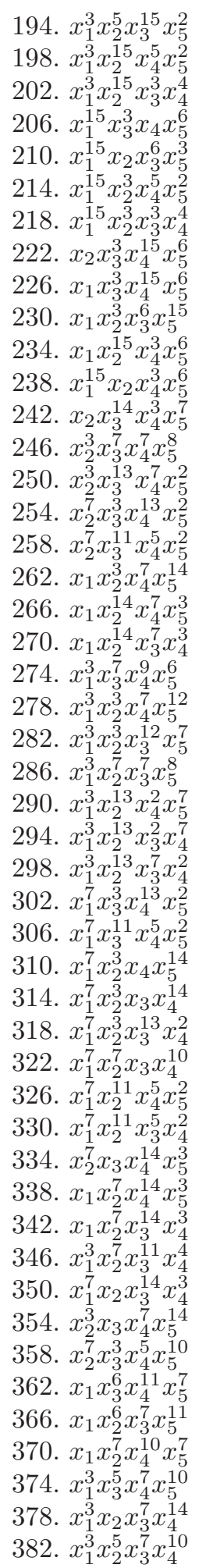 & 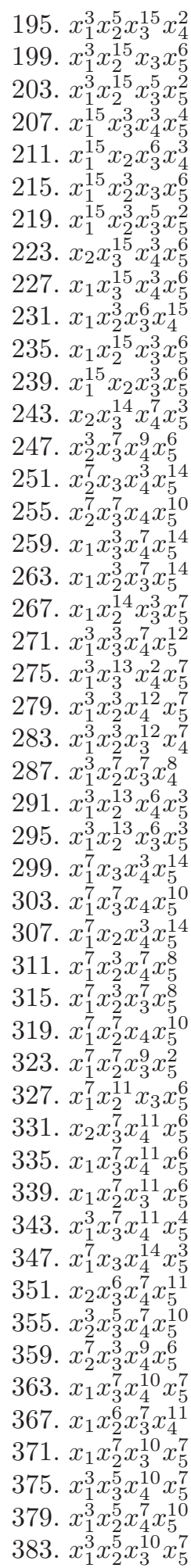 & 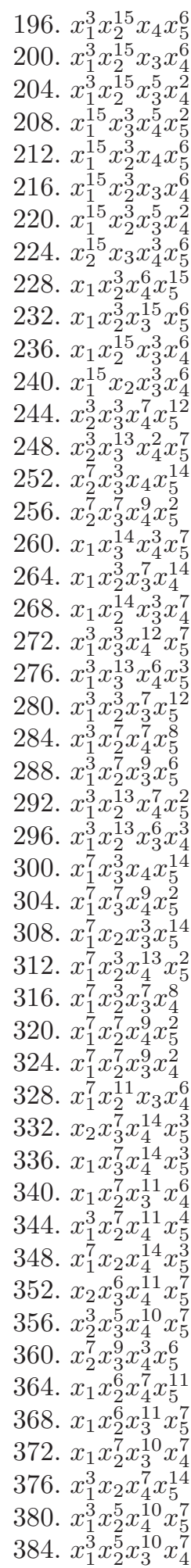 \\
\hline
\end{tabular}




\begin{tabular}{|c|c|c|c|}
\hline $\begin{array}{l}x_{1}^{7} x_{3} x_{4}^{10} x_{5}^{7} \\
x_{1}^{7} x_{2} x_{4}^{10} x_{5}^{7} \\
x_{1}^{7} x_{2}^{3} x_{4}^{9} x_{5}^{6} \\
x_{1}^{7} x_{2}^{3} x_{3}^{9} x_{4}^{6} \\
x_{2} x_{3}^{7} x_{4}^{7} x_{5}^{10} \\
x_{2}^{7} x_{3} x_{4}^{7} x_{5}^{10} \\
x_{1} x_{2}^{7} x_{3}^{7} x_{4}^{10} \\
x_{1}^{3} x_{2} x_{4}^{14} x_{5}^{7} \\
x_{1}^{3} x_{2}^{7} x_{3}^{5} x_{5}^{10} \\
x_{1}^{3} x_{2}^{13} x_{3}^{3} x_{4}^{6} \\
x_{1}^{7} x_{2} x_{3}^{7} x_{4}^{10} \\
x_{2}^{3} x_{3}^{5} x_{4}^{14} x_{5}^{3} \\
x_{2}^{7} x_{3}^{7} x_{4}^{8} x_{5}^{3} \\
x_{1}^{3} x_{3}^{5} x_{4}^{14} x_{5}^{3} \\
x_{1}^{3} x_{2}^{5} x_{4}^{14} x_{5}^{3} \\
x_{1}^{3} x_{2}^{5} x_{3}^{6} x_{4}^{11} \\
x_{1}^{3} x_{2}^{5} x_{3}^{14} x_{4}^{3} \\
x_{1}^{7} x_{3}^{7} x_{4}^{8} x_{5}^{3} \\
x_{1}^{7} x_{2}^{3} x_{4}^{3} x_{5}^{12} \\
x_{1}^{7} x_{2}^{3} x_{3}^{11} x_{5}^{4} \\
x_{1}^{7} x_{2}^{7} x_{3}^{8} x_{4}^{3} \\
x_{1}^{3} x_{3}^{3} x_{4}^{5} x_{5}^{14} \\
x_{1}^{3} x_{2}^{3} x_{3}^{5} x_{5}^{14} \\
x_{1}^{7} x_{3}^{7} x_{4}^{3} x_{5}^{8} \\
x_{2}^{3} x_{3}^{7} x_{4}^{3} x_{5}^{12} \\
x_{1}^{3} x_{2}^{7} x_{3}^{3} x_{4}^{12} \\
x_{2}^{3} x_{3}^{7} x_{4} x_{5}^{14} \\
x_{1} x_{3}^{3} x_{4}^{14} x_{5}^{7} \\
x_{1} x_{2}^{3} x_{3}^{14} x_{5}^{7} \\
x_{1} x^{7} x^{3} x_{14}\end{array}$ & $\begin{array}{l}\text { 386. } x_{1}^{7} x_{3}^{3} x_{4}^{5} x_{5}^{10} \\
\text { 390. } x_{1}^{7} x_{2} x_{3}^{10} x_{5}^{7} \\
\text { 394. } x_{1}^{7} x_{2}^{3} x_{3}^{5} x_{5}^{10} \\
\text { 398. } x_{1}^{7} x_{2}^{9} x_{4}^{3} x_{5}^{6} \\
\text { 402. } x_{2}^{3} x_{3} x_{4}^{14} x_{5}^{7} \\
\text { 406. } x_{1} x_{3}^{7} x_{4}^{7} x_{5}^{10} \\
\text { 410. } x_{1}^{3} x_{3} x_{4}^{14} x_{5}^{7} \\
\text { 414. } x_{1}^{3} x_{2} x_{3}^{14} x_{5}^{7} \\
\text { 418. } x_{1}^{3} x_{2}^{7} x_{3}^{5} x_{4}^{10} \\
\text { 422. } x_{1}^{7} x_{3} x_{4}^{7} x_{5}^{10} \\
\text { 426. } x_{2}^{3} x_{3}^{5} x_{4}^{3} x_{5}^{14} \\
\text { 430. } x_{2}^{7} x_{3} x_{4}^{11} x_{5}^{6} \\
\text { 434. } x_{1}^{3} x_{3}^{5} x_{4}^{3} x_{5}^{14} \\
\text { 438. } x_{1}^{3} x_{2}^{5} x_{4}^{3} x_{5}^{14} \\
\text { 442. } x_{1}^{3} x_{2}^{5} x_{3}^{3} x_{5}^{14} \\
\text { 446. } x_{1}^{3} x_{2}^{5} x_{3}^{11} x_{5}^{6} \\
\text { 450. } x_{1}^{7} x_{3} x_{4}^{11} x_{5}^{6} \\
\text { 454. } x_{1}^{7} x_{2} x_{4}^{11} x_{5}^{6} \\
\text { 458. } x_{1}^{7} x_{2}^{3} x_{4}^{11} x_{5}^{4} \\
\text { 462. } x_{1}^{7} x_{2}^{3} x_{3}^{11} x_{4}^{4} \\
\text { 466. } x_{2}^{3} x_{3}^{3} x_{4}^{5} x_{5}^{14} \\
\text { 470. } x_{1}^{3} x_{3}^{3} x_{4}^{13} x_{5}^{6} \\
\text { 474. } x_{1}^{3} x_{2}^{3} x_{3}^{5} x_{4}^{14} \\
\text { 478. } x_{1}^{7} x_{2}^{7} x_{4}^{3} x_{5}^{8} \\
\text { 482. } x_{1}^{3} x_{3}^{7} x_{4}^{3} x_{5}^{12} \\
\text { 486. } x_{2} x_{3}^{3} x_{4}^{14} x_{5}^{7} \\
\text { 490. } x_{2}^{3} x_{3}^{7} x_{4}^{13} x_{5}^{2} \\
\text { 494. } x_{1} x_{3}^{7} x_{4}^{3} x_{5}^{14} \\
\text { 498. } x_{1} x_{2}^{3} x_{3}^{14} x_{4}^{7} \\
502 . x_{1} x_{2}^{7} x_{3}^{3} x_{4}^{14} \\
506 . x_{1}^{3} x_{3}^{7} x_{4}^{13} x_{5}^{2} \\
510 . x_{1}^{3} x_{2}^{7} x_{3} x_{4}^{14} \\
514 . x_{1}^{7} x_{3}^{11} x_{4}^{3} x_{5}^{4} \\
518 . x_{1}^{7} x_{2}^{11} x_{4}^{3} x_{5}^{4}\end{array}$ & $\begin{array}{l}\text { 443. } x_{1} x_{2}^{2} x_{3}^{3} x_{4}^{4} \\
\text { 447. } x_{1}^{3} x_{2}^{5} x_{3}^{11} x_{4}^{6} \\
\text { 451. } x_{1}^{7} x_{3}^{3} x_{4}^{3} x_{5}^{12} \\
\text { 455. } x_{1}^{7} x_{2} x_{3}^{11} x_{5}^{6} \\
\text { 459. } x_{1}^{7} x_{2}^{3} x_{3}^{3} x_{5}^{12} \\
\text { 463. } x_{1}^{7} x_{2}^{7} x_{4}^{8} x_{5}^{3} \\
\text { 467. } x_{2}^{3} x_{3}^{3} x_{4}^{13} x_{5}^{6} \\
\text { 471. } x_{1}^{3} x_{2}^{3} x_{4}^{5} x_{5}^{14} \\
\text { 475. } x_{1}^{3} x_{2}^{3} x_{3}^{13} x_{5}^{6} \\
\text { 479. } x_{1}^{7} x_{2}^{7} x_{3}^{3} x_{5}^{8} \\
\text { 483. } x_{1}^{3} x_{2}^{7} x_{4}^{3} x_{5}^{12} \\
\text { 487. } x_{2} x_{3}^{7} x_{4}^{3} x_{5}^{14} \\
\text { 491. } x_{2}^{7} x_{3} x_{4}^{6} x_{5}^{11} \\
\text { 495. } x_{1} x_{3}^{7} x_{4}^{6} x_{5}^{11} \\
\text { 499. } x_{1} x_{2}^{7} x_{4}^{3} x_{5}^{14} \\
\text { 503. } x_{1} x_{2}^{7} x_{3}^{6} x_{5}^{11} \\
\text { 507. } x_{1}^{3} x_{2}^{7} x_{4} x_{5}^{14} \\
\text { 511. } x_{1}^{3} x_{2}^{7} x_{3}^{13} x_{5}^{2} \\
\text { 515. } x_{1}^{7} x_{2} x_{4}^{6} x_{5}^{11} \\
\text { 519. } x_{1}^{7} x_{2}^{11} x_{3}^{3} x_{5}^{4}\end{array}$ & $\begin{array}{l}\text { 448. } x_{1}^{3} \\
\text { 452. } x_{1}^{7} \\
\text { 456. } x_{1}^{7} \\
\text { 460. } x_{1}^{7} \\
\text { 464. } x_{1}^{7} \\
\text { 468. } x_{2}^{7} \\
\text { 472. } x_{1}^{3} \\
\text { 476. } x_{1}^{3} \\
\text { 480. } x_{1}^{7} \\
\text { 484. } x_{1}^{3} \\
\text { 488. } x_{2} \\
\text { 492. } x_{2}^{7} \\
\text { 496. } x_{1} \\
\text { 500. } x_{1} \\
\text { 504. } x_{1} \\
\text { 508. } x_{1}^{3} \\
512 \cdot x_{1}^{3} \\
516 \cdot x_{1}^{7} \\
520 . x_{1}^{7}\end{array}$ \\
\hline
\end{tabular}

\subsection{The admissible monomials of degree 25 in $P_{5}^{+}$.}

We have $B_{5}^{+}(25)=B_{5}^{+}(3,3,2,1) \cup \psi\left(B_{5}(10)\right)$, where $B_{5}^{+}(3,3,2,1)$ is the set of 440 monomials $b_{25, u}, 1 \leqslant u \leqslant 440$ :
1. $x_{1} x_{2} x_{3}^{2} x_{4}^{6} x_{5}^{15}$
2. $x_{1} x_{2} x_{3}^{2} x_{4}^{15} x_{5}^{6}$
5. $x_{1} x_{2} x_{3}^{15} x_{4}^{2} x_{5}^{6}$
6. $x_{1} x_{2} x_{3}^{15} x_{4}^{6} x_{5}^{2}$
3. $x_{1} x_{2} x_{3}^{6} x_{4}^{2} x_{5}^{15}$
7. $x_{1} x_{2}^{2} x_{3}^{3} x_{4}^{4} x_{5}^{15}$
4. $x_{1} x_{2} x_{3}^{6} x_{4}^{15} x_{5}^{2}$
9. $x_{1} x_{2}^{2} x_{3}^{4} x_{4}^{3} x_{5}^{15}$
10. $x_{1} x_{2}^{2} x_{3}^{4} x_{4}^{15} x_{5}^{3}$
11. $x_{1} x_{2}^{2} x_{3}^{15} x_{4}^{3} x_{5}^{4}$
8. $x_{1} x_{2}^{2} x_{3}^{3} x_{4}^{15} x_{5}^{4}$
13. $x_{1} x_{2}^{3} x_{3}^{2} x_{4}^{4} x_{5}^{15}$
14. $x_{1} x_{2}^{3} x_{3}^{2} x_{4}^{15} x_{5}^{4}$
15. $x_{1} x_{2}^{3} x_{3}^{15} x_{4}^{2} x_{5}^{4}$
12. $x_{1} x_{2}^{2} x_{3}^{15} x_{4}^{4} x_{5}^{3}$
17. $x_{1} x_{2}^{6} x_{3} x_{4}^{15} x_{5}^{2}$
18. $x_{1} x_{2}^{6} x_{3}^{15} x_{4} x_{5}^{2}$
19. $x_{1} x_{2}^{15} x_{3} x_{4}^{2} x_{5}^{6}$
16. $x_{1} x_{2}^{6} x_{3} x_{4}^{2} x_{5}^{15}$
21. $x_{1} x_{2}^{15} x_{3}^{2} x_{4}^{3} x_{5}^{4}$
22. $x_{1} x_{2}^{15} x_{3}^{2} x_{4}^{4} x_{5}^{3}$
23. $x_{1} x_{2}^{15} x_{3}^{3} x_{4}^{2} x_{5}^{4}$
20. $x_{1} x_{2}^{15} x_{3} x_{4}^{6} x_{5}^{2}$
25. $x_{1}^{3} x_{2} x_{3}^{2} x_{4}^{4} x_{5}^{15}$
29. $x_{1}^{3} x_{2} x_{3}^{15} x_{4}^{2} x_{5}^{4}$
26. $x_{1}^{3} x_{2} x_{3}^{2} x_{4}^{15} x_{5}^{4}$
27. $x_{1}^{3} x_{2} x_{3}^{4} x_{4}^{2} x_{5}^{15}$
24. $x_{1} x_{2}^{15} x_{3}^{6} x_{4} x_{5}^{2}$
30. $x_{1}^{3} x_{2} x_{3}^{15} x_{4}^{4} x_{5}^{2}$
31. $x_{1}^{3} x_{2}^{4} x_{3} x_{4}^{2} x_{5}^{15}$
28. $x_{1}^{3} x_{2} x_{3}^{4} x_{4}^{15} x_{5}^{2}$
33. $x_{1}^{3} x_{2}^{4} x_{3}^{15} x_{4} x_{5}^{2}$
34. $x_{1}^{3} x_{2}^{15} x_{3} x_{4}^{2} x_{5}^{4}$
35. $x_{1}^{3} x_{2}^{15} x_{3} x_{4}^{4} x_{5}^{2}$
32. $x_{1}^{3} x_{2}^{4} x_{3} x_{4}^{15} x_{5}^{2}$
37. $x_{1}^{15} x_{2} x_{3} x_{4}^{2} x_{5}^{6}$
38. $x_{1}^{15} x_{2} x_{3} x_{4}^{6} x_{5}^{2}$
39. $x_{1}^{15} x_{2} x_{3}^{2} x_{4}^{3} x_{5}^{4}$
36. $x_{1}^{3} x_{2}^{15} x_{3}^{4} x_{4} x_{5}^{2}$
40. $x_{1}^{15} x_{2} x_{3}^{2} x_{4}^{4} x_{5}^{3}$ 


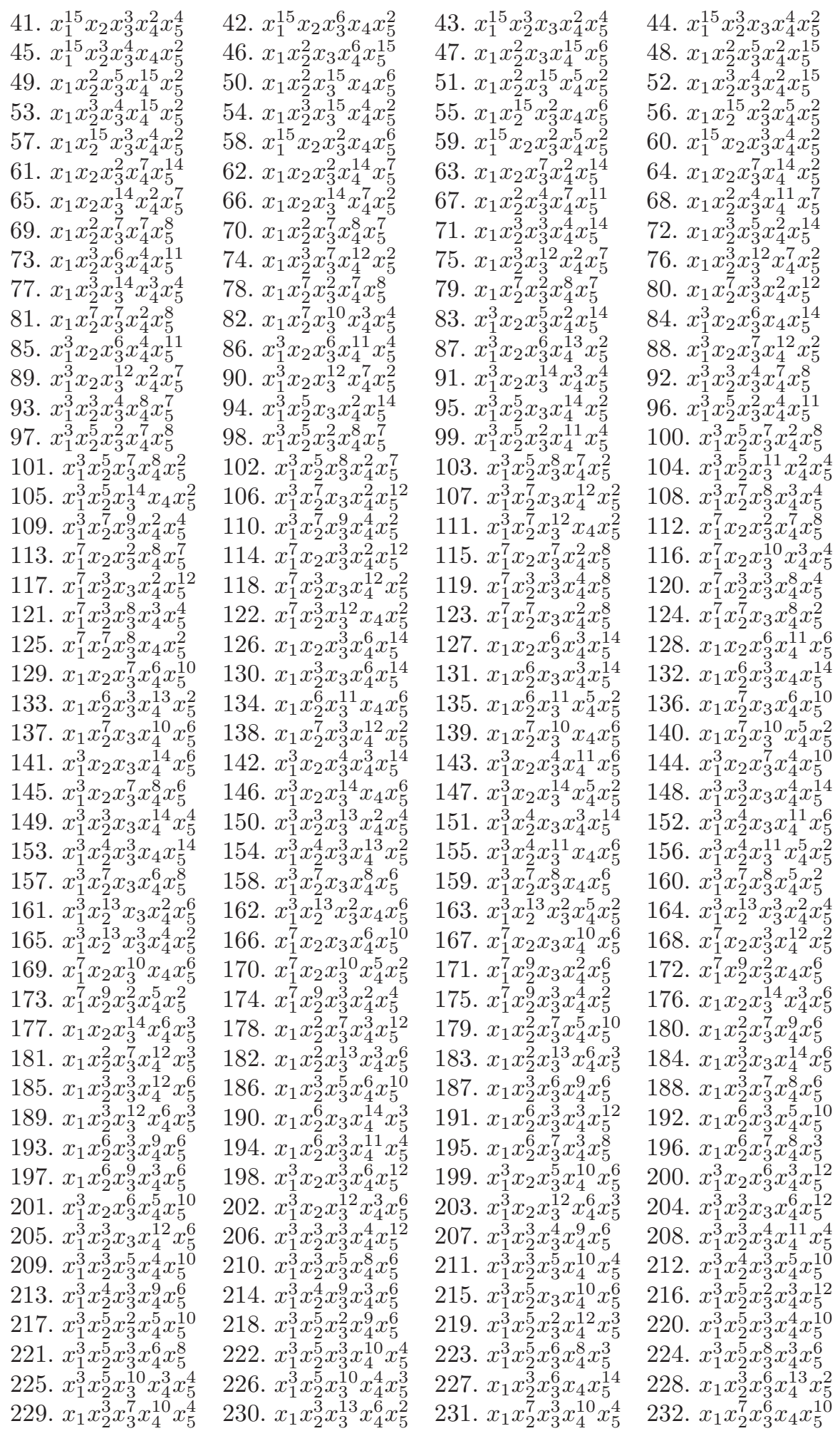




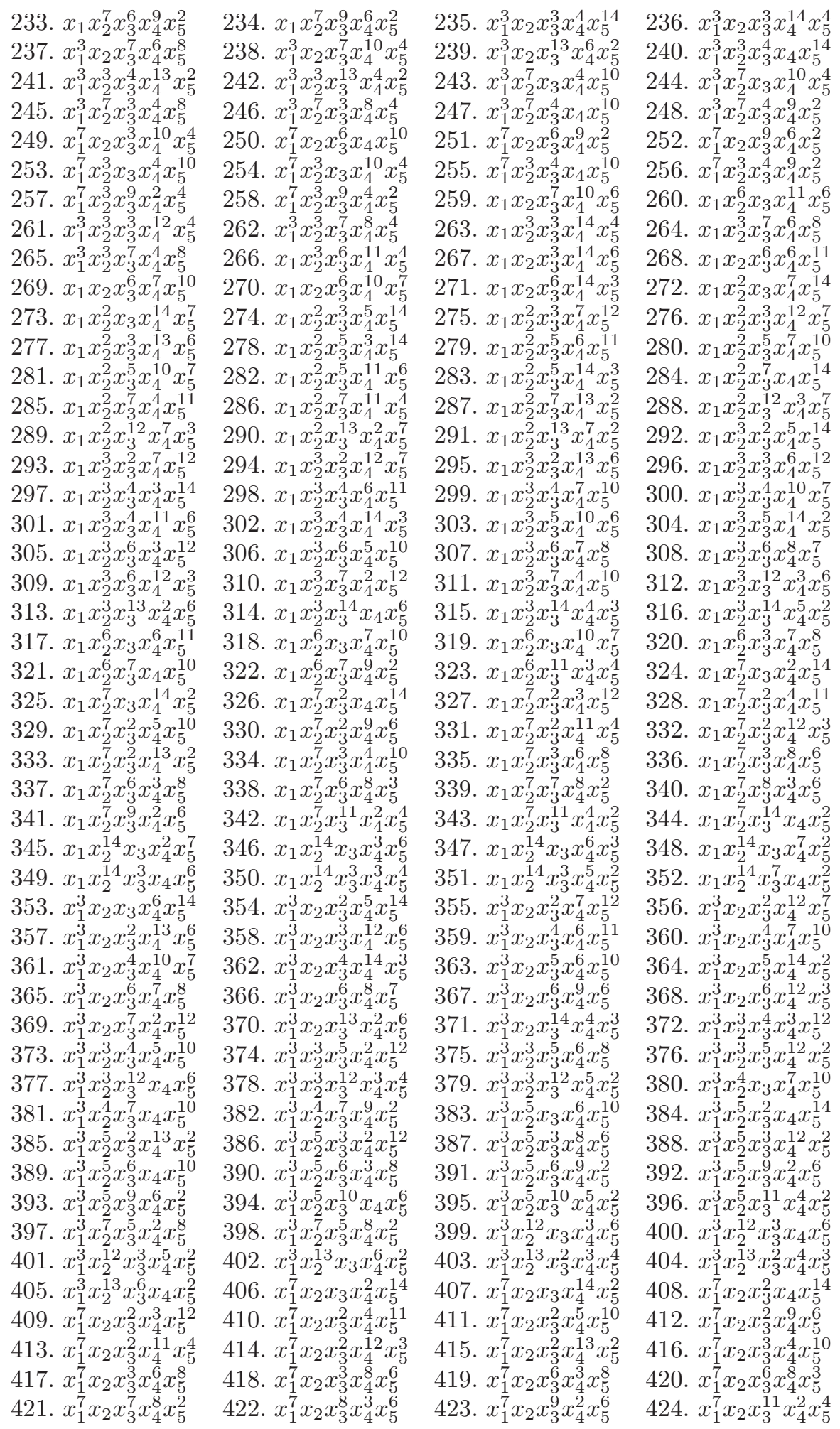



425. $x_{1}^{7} x_{2} x_{3}^{11} x_{4}^{4} x_{5}^{2}$
426. $x_{1}^{7} x_{2} x_{3}^{14} x_{4} x_{5}^{2}$
427. $x_{1}^{7} x_{2}^{3} x_{3} x_{4}^{6} x_{5}^{8}$
428. $x_{1}^{7} x_{2}^{3} x_{3} x_{4}^{8} x_{5}^{6}$
429. $x_{1}^{7} x_{2}^{3} x_{3}^{5} x_{4}^{2} x_{5}^{8}$
430. $x_{1}^{7} x_{2}^{3} x_{3}^{5} x_{4}^{8} x_{5}^{2}$
431. $x_{1}^{7} x_{2}^{3} x_{3}^{8} x_{4} x_{5}^{6}$
433. $x_{1}^{7} x_{2}^{8} x_{3} x_{4}^{3} x_{5}^{6}$
434. $x_{1}^{7} x_{2}^{8} x_{3}^{3} x_{4} x_{5}^{6}$
435. $x_{1}^{7} x_{2}^{8} x_{3}^{3} x_{4}^{5} x_{5}^{2}$
432. $x_{1}^{7} x_{2}^{3} x_{3}^{8} x_{4}^{5} x_{5}^{2}$
436. $x_{1}^{7} x_{2}^{9} x_{3} x_{4}^{6} x_{5}^{2}$
437. $x_{1}^{7} x_{2}^{9} x_{3}^{2} x_{4}^{3} x_{5}^{4}$
438. $x_{1}^{7} x_{2}^{11} x_{3} x_{4}^{2} x_{5}^{4}$
439. $x_{1}^{7} x_{2}^{11} x_{3} x_{4}^{4} x_{5}^{2}$
440. $x_{1}^{7} x_{2}^{11} x_{3}^{4} x_{4} x_{5}^{2}$

By a direct computation using the results in [26], we have

$$
\left|\Phi\left(B_{4}(3,3,2,1)\right)\right|=361 \text { and } \Phi\left(B_{4}(3,3,2,1)\right) \subset B_{5}(3,3,2,1) .
$$

If $\omega$ is a weight vector of degree 25 and $\omega \neq(3,3,2,1)$, then $B_{4}(\omega)=\emptyset$. If $s>1$, then $B_{4}\left(15.2^{s}-5\right)=\emptyset$. Hence, Conjecture 3.4 is true for $n=5$ and the degree $15.2^{s}-5$ with $s$ an arbitrary positive integer.

\section{ACKNOWLEDGMENT}

The first manuscript of this paper was written when the author was visiting the Vietnam Institute for Advanced Study in Mathematics (VIASM) from August to November 2017. He would like to thank the VIASM for financial support, convenient working condition and for kind hospitality.

The author was supported in part by the National Foundation for Science and Technology Development (NAFOSTED) of Viet Nam under the grant number 101.04-2017.05.

\section{REFERENCES}

1. J. M. Boardman, Modular representations on the homology of power of real projective space, in: M. C. Tangora (Ed.), Algebraic Topology, Oaxtepec, 1991, in: Contemp. Math., vol. 146, 1993, pp. 49-70.

2. R. R. Bruner, L. M. Hà and N. H. V. Hưng, On behavior of the algebraic transfer, Trans. Amer. Math. Soc. 357 (2005), 473-487.

3. D. P. Carlisle and R. M. W. Wood, The boundedness conjecture for the action of the Steenrod algebra on polynomials, in: N. Ray and G. Walker (ed.), Adams Memorial Symposium on Algebraic Topology 2, (Manchester, 1990), in: London Math. Soc. Lecture Notes Ser., Cambridge Univ. Press, Cambridge, vol. 176, 1992, pp. 203-216.

4. M. C. Crabb and J. R. Hubbuck, Representations of the homology of BV and the Steenrod algebra II, Algebraic Topology: new trend in localization and periodicity, Progr. Math. 136 (1996), 143-154.

5. T. W. Chen, Determination of $\operatorname{Ext}_{\mathcal{A}}^{5, *}(\mathbb{Z} / 2, \mathbb{Z} / 2)$, Topology Appl., 158 (2011), 660-689.

6. P. H. Chơn and L. M. Hà, On May spectral sequence and the algebraic transfer, Manuscripta Math. 138 (2012), 141-160.

7. P. H. Chơn and L. M. Hà, On the May spectral sequence and the algebraic transfer II, Topology Appl. 178 (2014), 372-383.

8. N. H. V. Hưng, The cohomology of the Steenrod algebra and representations of the general linear groups, Trans. Amer. Math. Soc. 357 (2005), 4065-4089.

9. N. H. V. Hưng and V. T. N. Quỳnh, The image of Singer's fourth transfer, C. R. Math. Acad. Sci. Paris 347 (2009), 1415-1418.

10. A. S. Janfada and R. M. W. Wood, The hit problem for symmetric polynomials over the Steenrod algebra, Math. Proc. Cambridge Philos. Soc.133 (2002), 295-303.

11. M. Kameko, Products of projective spaces as Steenrod modules, PhD. Thesis, The Johns Hopkins University, ProQuest LLC, Ann Arbor, MI, 1990. 29 pp.

12. W. H. Lin, $\operatorname{Ext}_{\mathcal{A}}^{4, *}(\mathbb{Z} / 2, \mathbb{Z} / 2)$ and $\operatorname{Ext}_{\mathcal{A}}^{5, *}(\mathbb{Z} / 2, \mathbb{Z} / 2)$, Topology Appl., 155 (2008), 459-496.

13. N. Minami, The iterated transfer analogue of the new doomsday conjecture, Trans. Amer. Math. Soc. 351 (1999), 2325-2351.

14. M. F. Mothebe, Generators of the polynomial algebra $\mathbb{F}_{2}\left[x_{1}, \ldots, x_{n}\right]$ as a module over the Steenrod algebra, PhD Thesis, The University of Manchester, 1997.

15. T. N. Nam, A-générateurs génériquess pour l'algèbre polynomiale, Adv. Math. 186 (2004), 334-362. 
16. T. N. Nam, Transfert algébrique et action du groupe linéaire sur les puissances divisées modulo 2, Ann. Inst. Fourier (Grenoble) 58 (2008), 1785-1837.

17. F. P. Peterson, Generators of $H^{*}\left(\mathbb{R} P^{\infty} \times \mathbb{R} P^{\infty}\right)$ as a module over the Steenrod algebra, Abstracts Amer. Math. Soc. No. 833 (1987), 55-89.

18. Đ. V. Phúc and N. Sum, On the generators of the polynomial algebra as a module over the Steenrod algebra, C. R. Acad. Sci. Paris, Ser. I 353 (2015), 1035-1040.

19. Đ. V. Phúc and N. Sum, On a minimal set of generators for the polynomial algebra of five variables as a module over the Steenrod algebra, Acta Math. Vietnam. 42 (2017), 149-162.

20. S. Priddy, On characterizing summands in the classifying space of a group, I, Amer. Jour. Math. 112 (1990), 737-748.

21. J. H. Silverman, Hit polynomials and the canonical antiautomorphism of the Steenrod algebra, Proc. Amer. Math. Soc. 123 (1995), 627-637.

22. J. H. Silverman and W. M. Singer, On the action of Steenrod squares on polynomial algebras II, J. Pure Appl. Algebra 98 (1995), 95-103.

23. W. M. Singer, The transfer in homological algebra, Math. Zeit. 202 (1989), 493-523.

24. W. M. Singer, On the action of the Steenrod squares on polynomial algebras, Proc. Amer. Math. Soc. 111 (1991), 577-583.

25. N. E. Steenrod and D. B. A. Epstein, Cohomology operations, Annals of Mathematics Studies 50, Princeton University Press, Princeton N.J (1962).

26. N. Sum, The negative answer to Kameko's conjecture on the hit problem, Adv. Math. 225 (2010), 2365-2390.

27. N. Sum, On the Peterson hit problem, Adv. Math. 274 (2015), 432-489.

28. N. Sum, On the determination of the Singer transfer, Vietnam Journal of Science, Technology and Engineering, 60(1) (2018), 3-16.

29. N. Sum, The squaring operation and the Singer algebraic tranfer, Preprint 2017, 42 pages; available online at https://arxiv.org/abs/1609.03006.

30. M. C. Tangora, On the cohomology of the Steenrod algebra, Math. Zeit. 116 (1970), 18-64.

31. N. K. Tín, The admissible monomial basis for the polynomial algebra of five variables in degree eleven, Quy Nhơn University Journal of Science, 6 (2012), 81-89.

32. N. K. Tín, The admissible monomial basis for the polynomial algebra of five variables in degree $2^{s+1}+2^{s}-5$, East-West J. of Mathematics, 16 (2014), 34-46.

33. N. K. Tín, On Singer's conjecture for the fifth algebraic transfer, Preprint 2016, 25 pages; available online at https://arxiv.org/abs/1609.02250.

34. N. K. Tín and N. Sum, Kameko's homomorphism and the algebraic transfer, C. R. Acad. Sci. Paris, Ser. I 354 (2016), 940-943.

35. G. Walker and R. M. W. Wood, Young tableaux and the Steenrod algebra, in: Proceedings of the International School and Conference in Algebraic Topology, Hà Nội 2004, Geom. Topol. Monogr., Geom. Topol. Publ., Coventry, vol. 11, 2007, pp. 379-397.

36. R. M. W. Wood, Steenrod squares of polynomials and the Peterson conjecture, Math. Proc. Cambriges Phil. Soc. 105 (1989), 307-309.

37. R. M. W. Wood, Problems in the Steenrod algebra, Bull. London Math. Soc. 30 (1998), 449517.

Department of Mathematics, Quy Nhơn University, 170 An Dương Vương, Quy Nhơn, Bình Định, Viet NAM

E-mail address: nguyensum@qnu.edu.vn 